\title{
LOCAL BLOW-UP OF STRATIFIED SETS UP TO BORDISM
}

BY

\author{
S. BUONCRISTIANO AND M. DEDÒ
}

\begin{abstract}
Homological obstructions are given, whose vanishing is a necessary and sufficient condition for the existence of a blow-up of an abstract prestratification $V$ along a 'locally top-dimensional' substratification $V^{\prime}$.
\end{abstract}

Introduction. Let us begin with an example. Let $W$ be the figure eight in the plane, in which we distinguish two strata, namely $O$ and $Y=W-O$. Let $\Sigma \mathbf{P}$ be the suspension on the real projective plane with strata $Z=A \cup B$ and $X=\Sigma \mathbf{P}-Z$, where $A$ and $B$ are the vertices of $\Sigma \mathbf{P}$. The product $V=W \times \Sigma \mathbf{P}$ has singularity $S V$ formed by the strata $O \times X, O \times Z, Y \times Z$. It is easy to see that $V$ does not admit a blow-up which is a smooth manifold, since the link of $Y \times Z$ in $V$ is the manifold $\mathbf{P}$ which does not bound. Thus we may study the following problem of local resolvability. Let $V^{\prime}=O \times \Sigma \mathbf{P}$ with strata $O \times X, O \times Z$ and let $N$ be a small neighbourhood of $V^{\prime}$ in $V ; N$ has singularity $S N$ in codimension one, while $N-V^{\prime}$ has singularity in codimension two. Is it possible to construct a blow-up $\hat{V} \rightarrow V$ which "coincides" with $V$ outside $N$ and such that the codimension of $S \hat{V}$ is equal to two? In the example which we are considering, $\hat{V}$ is easily obtained: it suffices to take $\hat{V}=\hat{W} \times \Sigma \mathbf{P}$, where $\hat{W}$ is a resolution of $W$ as indicated in Figure 1 .

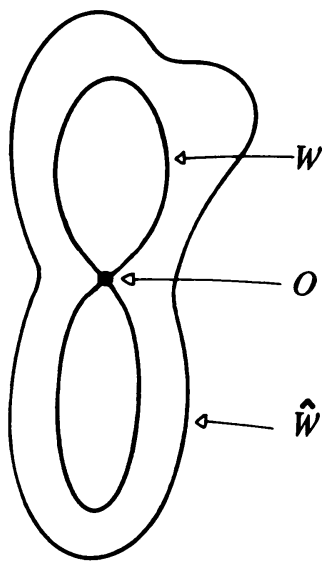

FiguRE 1

Received by the editors January 26, 1981.

1980 Mathematics Subject Classification. Primary 58A35; Secondary 57R12.

Key words and phrases. Stratified set, blow-up, bordism, characteristic numbers.

(c) 1982 American Mathematical Society $0002-9947 / 82 / 0000-0073 / \$ 07.75$ 
In this paper we study the problem of local resolution in the context of abstract prestratifications (according to Mather [8]). Let $V$ be such a stratified set and $V^{\prime} \subset V$ a substratification which is locally top-dimensional (Definition 2.1). Let $N=T\left(V^{\prime}, V\right)$ be the tubular neighbourhood of $V^{\prime}$ in $V$. Theorem 2.13 gives homological obstructions whose vanishing is a necessary and sufficient condition for the existence of a blow-up $\hat{V} \rightarrow V$ along $V^{\prime}$ (Definition 2.3) which replaces $N$ by $\hat{N}$ such that $\operatorname{dim} S \hat{N}=\operatorname{dim} S\left(N-V^{\prime}\right)$. Thus the problem is that of resolving the "higher" strata of $V^{\prime}$ up to a blow-up.

The paper is organised as follows.

In $\$ 1$ we recall those properties of stratified sets which will be used in the sequel. We prove that the bordism theory of stratified cycles is an ordinary homology theory with coefficients in $\mathbf{Z}_{2}$. Then we define auxiliary objects, namely stratified sets endowed with a compatible system of trivialisations of their tubular neighbourhoods. These objects are not without independent interest.

$\$ 2$ is dedicated to the definition of the obstructions and to the proof of Theorem 2.13.

In $\$ 3$ we briefly explain the difficulties which arise in trying to extend the previously developed theory to the case of $G$-stratified sets, where $G$ is one of the classical Lie groups $S O, U, S U, S p$. As one should expect, the vanishing of the obstructions is now a sufficient condition for the existence of $\hat{V}$ only in the presence of additional hypothesis on the homology of $V^{\prime}$.

\section{Stratified sets.}

Notation. [ ] will mean homology class, bordism class in the appropriate group, or fundamental class, depending on the context.

$A+B$ will be the disjoint union of the topological spaces $A$ and $B$.

Manifold will mean $C^{\infty}$ manifold of finite dimension.

Up to $§ 3$, (co)homology will be with $\mathbf{Z}_{2}$ coefficients.

Below we recall the definition and the basic properties of a (bounded) stratified set; for full details the reader is referred to $[8,7,17,14]$.

1.1 Definition. A stratified set is a triple $\{V, \chi, \tau\}$ satisfying the following axioms:

(A1) $V$ is a Hausdorff, locally compact, topological space with a countable basis for its topology. In $V$ there is given a closed subset $\dot{V}$.

(A2) $\chi$ is a family of locally closed subsets of $V$ and $V$ is the disjoint union of the members of $\chi$.

The members of $\chi$ are called the strata of $V$.

(A3) Each stratum $X$ of $V$ is a topological manifold (in the induced topology), possibly with boundary $\partial X$, provided with a smooth structure of class $C^{\infty} \cdot \dot{V}$ is the disjoint union of $\{\partial X, X \in \chi\}$.

(A4) The family $\chi$ is locally finite.

(A5) The family $\chi$ satisfies the axiom of the frontier: if $X, Y \in \chi$ and $Y \cap \bar{X} \neq \varnothing$, then $Y \subseteq \bar{X}$; if $Y \subseteq \bar{X}$ and $Y \neq X$, we write $Y<X$.

(A6) $\tau$ is a triple $\left\{\left\{T_{X}\right\},\left\{\pi_{X}\right\},\left\{\rho_{X}\right\}\right\}$, where, for each $X \in \chi, T_{X}$ is an open neighbourhood of $X$ in $V, \pi_{X}$ is a continuous retraction of $T_{X}$ onto $X, \pi_{X} \mid T_{X} \cap \dot{V}$ is 
a continuous retraction of $T_{X} \cap \dot{V}$ onto $\partial X$ and $\rho_{X}$ is a continuous function $\rho_{X}$ : $T_{X} \rightarrow[0, \infty)$.

$T_{X}$ is called the tubular neighbourhood of $X, \pi_{X}$ the local retraction of $T_{X}$ onto $X$ and $\rho_{X}$ the tubular function of $X$.

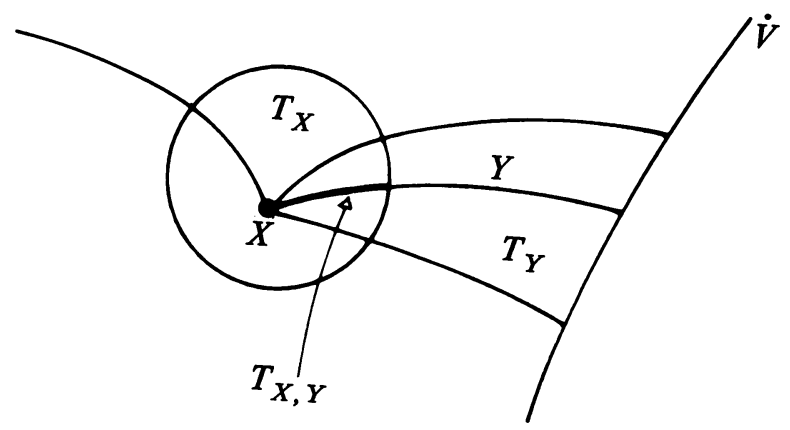

Figure 2

(A7) $X=\left\{v \in T_{X}: \rho_{X}(v)=0\right\}$. If $X$ and $Y$ are any strata, we let $T_{X, Y}=T_{X} \cap Y$, $\pi_{X, Y}=\pi_{X} \mid T_{X, Y}$ and $\rho_{X, Y}=\rho_{X} \mid T_{X, Y}$ (see Figure 2). Then $\pi_{X, Y}$ is a map of $T_{X, Y}$ onto $X$ and $\rho_{X, Y}$ is a map of $T_{X, Y}$ onto $(0, \infty)$. Note that $T_{X, Y} \cap \dot{V}=T_{X} \cap \partial Y$.

(A8) For any strata $X<Y$, the maps

$$
\begin{gathered}
\left(\pi_{X, Y}, \rho_{X, Y}\right): T_{X, Y} \rightarrow X \times(0, \infty), \\
\left(\pi_{X, Y}\left|, \rho_{X, Y}\right|\right): T_{X, Y} \cap \dot{V} \rightarrow \partial X \times(0, \infty)
\end{gathered}
$$

are smooth submersions.

(A9) For any strata $X, Y, Z$ we have

$$
\begin{aligned}
& \pi_{X, Y} \pi_{Y, Z}(v)=\pi_{X, Z}(v), \\
& \rho_{X, Y} \pi_{Y, Z}(v)=\rho_{X, Z}(v)
\end{aligned}
$$

whenever both sides of these equations are defined, i.e. whenever $v \in T_{Y, Z}$ and $\pi_{Y, Z}(v) \in T_{X, Y}$.

(A10) If $X, Y$ are strata and $T_{X, Y} \neq \varnothing$, then $X<Y$.

(A11) If $X, Y$ are strata and $T_{X} \cap T_{Y} \neq \varnothing$, then $X$ and $Y$ are comparable, i.e. one of the following holds: $X<Y, Y<X$ or $X=Y$.

We say that $(V, \chi, \tau)$ is a stratified set bounded by $\dot{V}$. If $\dot{V}=\varnothing, V$ is an unbounded stratified set with $\dot{\chi}=\{\partial X, X \in \chi\}$ and $\dot{\tau}=\left\{\left\{T_{X} \cap \dot{V}\right\},\left\{\pi_{X} \mid\right\},\left\{\rho_{X} \mid\right\}\right\}$.

We shall avoid mentioning $\dot{V}$, when this is obvious from the context.

Let $(V, \chi, \tau)$ be a compact stratified set. The cone $(c V, c \chi, c \tau)$ is defined as the mapping cylinder stratified set of the constant map $f: V \rightarrow\{0\}$.

The open cone $\left(c \stackrel{\circ}{V}, c^{\circ} \chi, c^{\circ} \tau\right)$ is the open mapping cylinder $c V=V \times[0, \infty) /(x, 0)$ $\sim\left(x^{\prime}, 0\right)$ with an obvious stratification and structure. In particular we have the

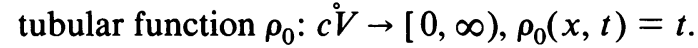

A subset $\chi^{\prime}$ of $\chi$ is a substratification of $\chi$ if, for every pair $(X, Y) \in \chi^{\prime} \times \chi$, $Y<X$ implies $Y \in \chi^{\prime}$. 
Then $V^{\prime}=\cup_{X \in \chi^{\prime}} X$ is a closed subspace of $V$ and is a stratified set with tubular neighbourhoods

$$
\tau^{\prime}=\left\{\left\{T_{X} \cap V^{\prime}\right\},\left\{\pi_{X} \mid T_{X} \cap V^{\prime}\right\},\left\{\rho_{X} \mid T_{X} \cap V^{\prime}\right\}, X \in \chi^{\prime}\right\}
$$

$V^{\prime}$ will be called a substratified set of $V$.

If $X$ is a stratum, then $\bar{X}=\cup_{Y \leqslant X} Y$ is a substratified set of $V$; more generally, for any subset $\zeta$ of $\chi$, we have a substratified set $\bar{\zeta}=\{Y \in \chi: Y \leqslant X \in \zeta\}$, called the closure of $\zeta$.

A stratum $X$ of $\chi$ is principal if there is no $Y \in \chi$ such that $X<Y$.

If $X \in \chi$, the frontier $\mathscr{F} X$ is the substratified set $\mathscr{F} X=\{Y \in \chi: Y<X\}$.

The $i$-skeleton $\chi^{(i)}=\{X \in \chi: \operatorname{dim} X \leqslant i\}$ is a substratified set of $V$.

We define the dimension of $V$ as the integer $\operatorname{dim} V=\max \{\operatorname{dim} X, X \in \chi\}$.

The depth of $V$ is the integer $d$ such that, if $n$ is the dimension of $V$, then $\chi^{(n-d)} \neq\{\varnothing\}$, while $\chi^{(n-h)}=\{\varnothing\}$ for $h>d$. Let $W$ be a subset of $V$ and suppose that $W$ itself is a stratified set. We say that $W$ is a restriction of $V$ if:

(a) for each stratum $X$ of $W$ there is a stratum $X^{\prime}$ of $V$ such that $X$ is a framed $C^{\infty}$ proper submanifold of $X^{\prime}$;

(b) $T_{X}=T_{X^{\prime} \cap W} ; \pi_{X}=\pi_{X^{\prime}}\left|T_{X} ; \rho_{X}=\rho_{X^{\prime}}\right| T_{X}$.

Let $X \in \chi$. Consider $V_{X}=\rho_{X}^{-1}[0,1]$ and $\dot{V}_{X}=\rho_{X}^{-1}(1) . V_{X}$ will be referred to as the closed tubular neighbourhood of $X$ in $V . V_{X}$ is a stratified set with strata $\left\{V_{X} \cap Y\right.$, $X \leqslant Y\}$ bounded by $\dot{V}_{X} \cup\left(V_{X} \cap \dot{V}\right)$.

If $Y>X$, the tubular neighbourhood $T_{Y}^{\prime}$ and the projections $\pi_{Y}^{\prime}$ are obtained by restricting the corresponding $T_{Y}$ and $\pi_{Y}$; the tubular functions $\rho_{Y}^{\prime}$ are the restrictions of the $\rho_{Y}$; for $Y=X$, we define $T_{X}^{\prime}=V_{X}-\dot{V}_{X}, \pi_{X}^{\prime}=\pi_{X} \mid$ and we need to reparametrize $\rho_{X}$ by the formula $\rho_{X}^{\prime}(v)=\operatorname{tang}\left(\rho_{X}(v) \cdot \pi / 2\right)$.

More generally, let $V^{\prime} \subset V$ be a substratified set. We call $T\left(V^{\prime}, V\right)=\cup_{X \in \chi^{\prime}} V_{X}$ the closed tubular neighbourhood of $V^{\prime}$ in $V$.

The exterior of $V^{\prime}$ in $V$ is defined by $E\left(V^{\prime}, V\right)=\overline{V-T\left(V^{\prime}, V\right)}$.

Similarly to the case $V^{\prime}=X$ above, one may define a structure of stratified sets on $T\left(V^{\prime}, V\right)$ and $E\left(V^{\prime}, V\right)$.

Let $\dot{T}\left(V^{\prime}, V\right)$ be the topological frontier of $T\left(V^{\prime}, V\right)$; let $\underline{X}=E(\mathscr{F} X, \bar{X})$ and $V_{\underline{X}}=\pi_{X}^{-1}(\underline{X})$. Then $V=\cup_{X \in X} V_{\underline{X}}$. Using this decomposition of $V$ and the stratified sets $V_{X}, \dot{V}_{X}$ defined above, one is able to prove the following:

(a) $\dot{T}\left(V^{\prime}, V\right)$ has a structure of a stratified set which is a restriction of $(V, \chi, \tau)$; $T\left(V^{\prime}, V\right)$ is bounded by $\dot{T}\left(V^{\prime}, V\right) \cup T\left(V^{\prime} \cap \dot{V}, \dot{V}\right)$.

(b) $T\left(V^{\prime}, V\right)$ is the mapping cylinder of a projection $\dot{\pi}: \dot{T}\left(V^{\prime}, V\right) \rightarrow V^{\prime}$.

For a proof the reader is referred to [17, 2.9].

Similarly $E\left(V^{\prime}, V\right)$ is a stratified set bounded by

$$
\dot{E}\left(V^{\prime}, V\right)=\dot{T}\left(V^{\prime}, V\right) \cup E\left(V^{\prime} \cap \dot{V}, \dot{V}\right)
$$

When there is no possibility of misunderstanding, we shall write $T_{V^{\prime}}$ and $E_{V^{\prime}}$ instead of $T\left(V^{\prime}, V\right)$ and $E\left(V^{\prime}, V\right)$.

Another important property of $V$ which we shall use is the following: $V$ has the homotopy type of a finite dimensional $\mathrm{CW}$-complex $[17,2.8]$. 
Finally, we call isomorphism of stratified sets a homeomorphism $f: V \rightarrow V^{\prime}$ such that:

(a) to each $X \in \chi$ there corresponds a $X^{\prime} \in \chi^{\prime}$ such that $f$ restricts to a diffeomorphism between $T_{X, Y}$ and $T_{X^{\prime}, Y^{\prime}}$, for all $X \leqslant Y$;

(b) $f \pi_{X}(v)=\pi_{X^{\prime}} f(v)$;

(c) $\rho_{X^{\prime}} f(v)=\rho_{X}(v)$.

Homology via stratified sets. Let $V$ be a compact stratified set of dimension $n$. The $(n-1)$-skeleton of $V$ will be referred to as the singularity of $V$, written $S V$.

1.2 Definition. A closed stratified $n$-cycle is a (necessarily unbounded) compact stratified set $V$ of dimension $n$ such that:

(a) $V=\overline{V-S V}$;

(b) $V-S V$ is an $n$-dimensional manifold without boundary;

(c) $\operatorname{dim} S V<n-1$.

Let us consider a pair $(V, \partial V)$ with $V$ a compact stratified set bounded by $\dot{V}$ and $\dot{V} \subset \partial V \subset V$; assume that $\partial V-\dot{V}$ is a substratified set of $V$. Then $\partial V$ has a structure of an unbounded stratified set induced by the structure of $\dot{V}$ and that of $\partial V-\dot{V}$.

1.3 Definition. A pair $(V, \partial V)$ as above is a relative stratified $n$-cycle (with boundary $\partial V$ ) if:

(a) $V=\overline{V-S V}$

(b) $V-S V$ is an $n$-dimensional manifold with boundary $\partial V-S V$;

(c) $\partial V$ is a closed stratified $(n-1)$-cycle with $S(\partial V)=S V \cap \partial V$;

(d) $\operatorname{dim} S V<n-1$.

Of course $\partial V$ may be empty, and in this case the above definition coincides with 1.2 .

Stratified cycles satisfy the following two easy but important properties.

$(\mathcal{P})$ Let $I=[0,1]$ with the obvious structure of stratified set bounded by $I$; then $V \times I$ may be given the structure of a relative stratified cycle with boundary $V \times \dot{I} \cup \partial V \times I$.

(9) Let $V, W$ be stratified $n$-cycles; $\partial V=V^{\prime} \cup V^{\prime \prime}$ with $V^{\prime}$ and $V^{\prime \prime}$ relative stratified $(n-1)$-cycles and $V^{\prime} \cap V^{\prime \prime}=\partial V^{\prime}=\partial V^{\prime \prime}$ and similarly for $\partial W=W^{\prime} U$ $W^{\prime \prime}$. If $h: V^{\prime} \rightarrow W^{\prime}$ is an isomorphism, then the union $U=V \cup_{h} W$ may be given the structure of a stratified $n$-cycle with boundary $\partial U=V^{\prime \prime} \cup_{h} W^{\prime \prime}$.

Using the above properties, the bordism group of stratified $n$-cycles in a topological pair $(A, B)$ may be defined in the usual way [5, §5]. It will be written $H_{n}^{\chi}(A, B)$. This is a functor on the category of topological pairs and continuous maps and there is a natural degree -1 transformation $\partial^{x}: H_{n}^{\chi}(A, B) \rightarrow H_{n-1}^{\chi}(B, \varnothing)$ obtained by restriction to the boundary.

1.4 THEOREM. $\left\{H_{n}^{\chi}(A, B), \partial^{\chi}\right\}$ is an ordinary homology theory with $\mathbf{Z}_{2}$ coefficients.

Proof. The homotopy axiom and the exactness axiom are proved much in the

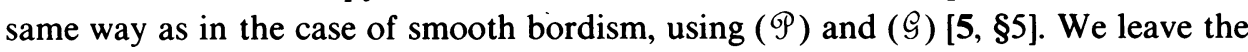
easy details to the reader. In order to prove the Mayer-Vietoris axiom we need the following definitions and lemma.

A closed subset $P$ of the stratified $n$-set $V$ is controlled if $P \cap T_{X}=\pi_{X}^{-1}(P \cap X)$ for $X \in \chi$. 
Let $f: V \rightarrow A$ be a continuous map from the stratified set $V$ to the topological space $A ; f$ is said to be controlled if $f \pi_{X}=f$ on $T_{X}$, for each $X \in \chi$.

In particular, if $f$ is controlled and $C$ is a closed subset of $A$, then $f^{-1}(C)$ is a controlled subset of $V$.

Let $P$ and $Q$ be two closed disjoint subsets of the stratified $n$-set $V$ and let $W$ be an $(n-1)$-dimensional restriction of $V$. Assume $V$ is connected. We say that $W$ separates $P$ and $Q$ if $V-W$ consists of two connected components $V_{P}$ and $V_{Q}$ such that $P \subset V_{P}$ and $Q \subset V_{Q}$. The definition extends immediately to a general $V$ componentwise.

1.5 Lemma. Let $P$ and $Q$ be disjoint controlled subsets of the stratified relative $n$-cycle $V$. There exists a restriction $W$ of $V$ such that $W$ separates $P$ and $Q$. Moreover, if $W^{\prime} \subset \partial V$ separates $P \cap \partial V$ and $Q \cap \partial V$, we may choose $W$ such that $W \cap \partial V=W^{\prime}$.

Proof. By induction on the depth $d$ of $V$. If $d=0, V$ is a manifold and the proposition is proved in $[5,3.1$, p. 10].

Let $V$ have depth $d$ and assume the proposition true for stratified cycles of depth $<d$. For simplicity assume that there is only one stratum $X$ in $X^{(d)}$; otherwise one applies the same argument simultaneously to all the strata in $X^{(d)}$. Assume also $\partial V=\varnothing$.

$X$ is an unbounded compact manifold and thus (by the case $d=0$ ) there exists a submanifold $W(X)$ of $X$ such that $W(X)$ separates $P \cap X$ and $Q \cap X$. Consider the exterior $E(X, V) . E(X, V)$ is a stratified set bounded by $\dot{V}_{X}=\rho_{X}^{-1}[0,1]$; the submersion conditions for $\pi_{X}$ imply that $W(\dot{E})=\pi_{X}^{-1}(W(X)) \cap \dot{V}_{X}$ is a stratified set restriction of $\dot{V}_{X}$.

Now $E(X, V)$ has depth $<d, P \cap E(X, V)$ and $Q \cap E(X, V)$ are controlled subsets of $E(X, V)$ and $W(\dot{E})$ separates $P \cap \dot{E}(X, V)$ and $Q \cap \dot{E}(X, V)$. Thus, by the inductive hypothesis, $W(\dot{E})$ extends to $W(E) \subset E(X, V)$ separating $P \cap$ $E(X, V)$ and $Q \cap E(X, V)$. Finally the required $W$ is given by $W(E) \cup \pi_{X}^{-1}(W(X))$ $\cap V_{X}$, the union being taken along $W(\dot{E})$. It is clear now that the same arguments allow one to deal with the case $\partial V \neq \varnothing$.

Proof of 1.4 (COntinuation). Now we use the lemma to show that the Mayer-Vietoris axiom is satisfied. Let $f: V \rightarrow A$ be a continuous map from the stratified set $V$ to the topological space $A$. Using the mapping cylinder structure of $T_{X}$ and the H.E.P., it is easy to prove (by induction on the depth of $V$ ) that $f$ is homotopic to a controlled map; moreover, if $f_{0}$ and $f_{1}$ are controlled and homotopic, then there exists a controlled homotopy joining $f_{0}$ to $f_{1}$.

Now assume that $V$ is a closed stratified $n$-cycle and $A=A_{1} \cup A_{2}$ with $A_{i}$ open in $A$, so that we have an element $[f: V \rightarrow A] \in H_{n}^{\chi}(A)$. We seek to define a Mayer-Vietoris homomorphism $\Delta: H_{n}^{\chi}(A) \rightarrow H_{n-1}^{\chi}\left(A_{1} \cap A_{2}\right)$. Up to homotopy, assume $f$ is controlled; then $P=f^{-1}\left(A-A_{1}\right)$ and $Q=f^{-1}\left(A-A_{2}\right)$ are disjoint (closed) controlled subsets of $V$. If $W$ is as in the lemma we define $\Delta[f: V \rightarrow A]=[f: W \rightarrow A]$.

By relativising the above construction in an obvious way, one sees that $\Delta$ is a well-defined homomorphism. At this point the Mayer-Vietoris axiom follows from standard bordism-type arguments. Finally, the dimension axiom is an immediate consequence of the following property of stratified cycles. 
(e) cone construction. If $V$ is a closed stratified cycle of positive dimension, $c V$ is a relative cycle with boundary $V$.

This completes the proof of the theorem.

Trivialised sets. Roughly speaking, these are stratified sets in which every tubular neighbourhood is a fibre bundle provided with a trivialisation. The system of trivialisations is required to be "compatible" with the projections and the tubular functions in the sense explained below. An alternative (equivalent) definition of a trivialised set is sketched at the end of this section.

1.6 Definition. A trivial set is a stratified set $(V, \chi, \tau)$ such that, for each $X \in \chi$ there is given an open cone $D(X)$ and a homeomorphism $h_{X}: T_{X} \rightarrow X \times D(X)$, called trivialisation.

1.7 Definition. A trivialised set is a trivial set $\left(V, \chi, \tau,\{D(X)\},\left\{h_{X}\right\}\right)$ satisfying the following compatibility conditions.

If $X \leqslant Y$, then $h_{X}$ restricts to a diffeomorphism between $T_{X, Y}$ and $X \times D(X)_{Y}$, with $D(X)_{Y}$ a stratum of $D(X) ; D(X)$ is in turn a trivial set with strata $\left\{D(X)_{Y}\right.$ : $X \leqslant Y\}$ and $D(Y)=D\left(D(X)_{Y}\right)$ (compatibility of duals); $h_{X}\left(T_{X} \cap T_{Y}\right)=X \times$ $T_{D(X)_{Y}}$.

On $T_{X} \cap T_{Y}$ we have

$$
\begin{gathered}
\left(1_{X} \times \pi_{D(X)_{Y}}\right) h_{X}=h_{X} \pi_{Y} \\
\left(1_{X} \times h_{D(X)_{Y}}\right) h_{x}=\left(h_{X} \times 1_{D(Y)}\right) h_{Y}
\end{gathered}
$$

(compatibility of trivialisations with projections: see diagram $\left.(1)_{V}\right)$ and

$$
\rho_{D(X)_{Y}} p_{2} h_{X}=\rho_{Y} ; \quad \rho_{0} p_{2} h_{D(X)_{Y}}=\rho_{D(X)_{Y}}
$$

(compatibility of trivialisations with tubular functions: see diagram $(2)_{V}$ ); here $p_{2}$ is the projection on the second factor and $\rho_{0}$ is the tubular function of $D(Y)$.

$$
\begin{aligned}
& \begin{array}{lll}
T_{X, Y} & \overrightarrow{h_{X}} & X \times D(X)_{Y} \\
\uparrow \pi_{Y} & & \uparrow 1_{X} \times \pi_{D(X)_{Y}}
\end{array}
\end{aligned}
$$

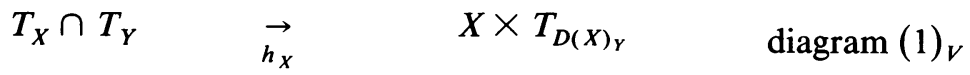

$$
\begin{aligned}
& \downarrow h_{Y} \quad \downarrow 1_{X} \times h_{D(X)_{Y}} \\
& T_{X, Y} \times D(Y) \quad \underset{h_{X} \times 1_{D(Y)}}{\rightarrow_{(1)}} \quad X \times D(X)_{Y} \times D(Y) \\
& X \times T_{D(X)_{Y}} \quad \stackrel{\leftarrow}{h_{X}} \quad T_{X} \cap T_{Y} \\
& \downarrow p_{2} \\
& T_{D(X)_{Y}} \quad \underset{\rho_{D(X)_{Y}}}{\rightarrow} \quad[0, \infty) \\
& \downarrow h_{D(X)_{Y}} \quad \uparrow \rho_{0} \\
& D(X)_{Y} \times D(Y) \quad \underset{p_{2}}{\rightarrow} \quad D(Y)
\end{aligned}
$$


If $D(X)$ is the open cone on $L(X)$, then $L(X)$ is referred to as the link of $X$. We identify $L(X)$ with $L(X) \times\{1\} \subset D(X)$ and write $C(X)$ for the cone $c L(X)$. Thus $h_{x}$ restricts to a map $V_{X}, \dot{V}_{X} \rightarrow X \times C(X), X \times L(X)$. When there is possibility of confusion, we shall write $L(X, V), D(X, V), C(X, V)$ instead of $L(X), D(X), C(X)$.

All terminology and notation introduced for stratified sets translates immediately into the context of trivialised sets. Below we sketch the modifications deriving from the additional structure present in a trivialised set.

The cone and the open cone over a trivialised set are trivialised sets in a natural way. Similarly for a substratified set of a trivialised set. If $W \subset V$ are trivialised sets, we say that $W$ is a restriction of $V$ if $W$ is a restriction of $V$ as stratified sets (i.e. (a) and (b) above hold) and (c) $L(X, V)=L\left(X^{\prime}, V\right) ; h_{X}=h_{X^{\prime}} \mid T_{X}$ (we are using previous notation).

Let $V, V^{\prime}$ be trivialised sets and $f: V \rightarrow V^{\prime}$ be an isomorphism of stratified sets. We say that $f$ is an isomorphism of trivialised sets if $f$ induces an isomorphism of diagrams between the various diagrams $(1)_{V},(2)_{V}$ and the corresponding $(1)_{V^{\prime}},(2)_{V^{\prime}}$. There is a bordism group $\bar{H}_{n}^{\chi}(A, B)$ defined in the same way as $H_{n}^{\chi}(A, B)$ but using trivialised $n$-cycles rather than only stratified $n$-cycles. The proof of the following theorem is thoroughly similar to that of Theorem 1.4.

Let $\bar{\partial}^{x}: \bar{H}_{n}^{\chi}(A, B) \rightarrow \bar{H}_{n-1}^{x}(B, \varnothing)$ be the natural transformation induced by restriction to the boundary.

1.8 THEOREM. $\left\{\bar{H}_{n}^{x}(A, B), \bar{\partial}^{x}\right\}$ is an ordinary homology theory with coefficients in $\mathbf{Z}_{2}$.

The pinched product $\underset{\sim}{\times}$. Let $(W, \chi, \tau)$ be a stratified set and $M$ a closed manifold. Consider the cone $c M$; the product $V=W \times c M$ is a stratified set (possibly after straightening the corner if $\dot{V} \neq \varnothing)$. Define $W \underset{\sim}{\times} c M=\cup_{X} \bar{V}_{X}$ and $W \underset{\sim}{\times} M=$ $\cup_{X} \overline{\dot{V}}_{X}$, where $X$ varies over the top-dimensional strata of $W$ and $\bar{V}_{X}=V_{X} \tilde{\cup} \mathscr{F} X$, $\dot{V}_{X}=\dot{V}_{X} \cup \mathcal{F} X . W \underset{\sim}{\times} c M$ and $W \underset{\sim}{\times} M$ are easily seen to be stratified sets (trivialised if $W$ is trivialised) with structure induced from $W \times c M$ by intersection (see Figure 3).

Note that, topologically, $W \underset{\sim}{\times} c M=W \times c M /(x, y) \sim\left(x, y^{\prime}\right)$ if $\left(x, y, y^{\prime}\right) \in$ $S W \times c M \times c M$, and similarly for $W \underset{\sim}{\times} M$. The following proposition follows easily from definitions.

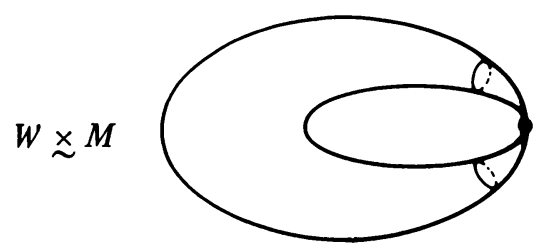

$W$

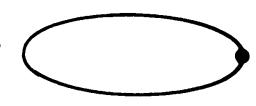

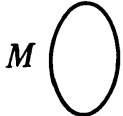

Figure 3 
1.9 Proposition. Let $\left(V, \chi, \tau,\left\{h_{X}\right\}\right)$ be a trivialised set and $X \in \chi$. Then $h_{X}$ induces isomorphisms of trivialised sets $\bar{V}_{X} \cong \bar{X} \underset{\sim}{\times} C(X, V)$ and $\bar{V}_{X} \cong \bar{X} \underset{\sim}{\times} L(X, V)$.

In view of the above proposition, we may confuse $\bar{V}_{X}$ and $\overline{\dot{V}}_{X}$ with $\bar{X} \underset{\sim}{ } \underset{ }{ } C(X)$ and $\bar{X} \underset{\tilde{N}}{\times} L(X)$ respectively and $V_{X}, \dot{V}_{X}$ with $\underline{X} \times C(X), \underline{X} \times L(X)$ respectively.

Note that, if $V$ is a cycle, $\bar{V} \times M$ is a relative cycle with boundary $\partial(V \times M)=$ $\partial V \underset{\sim}{\times} M$ and, if $\operatorname{dim} M>0, V \underset{\sim}{\times} c M$ is also a relative cycle with boundary

$$
\partial(V \underset{\sim}{\times} c M)=\partial V \underset{\sim}{\times} c M \cup V \underset{\sim}{\times} M
$$

the union being taken along $\partial V \underset{\sim}{\times} M$.

The alternative definition of a trivialised set. Here we limit ourselves to sketching the pattern and the main points of the definition, leaving the reader to complete the easy details.

A trivialised set consists of a stratified set (the underlying stratified set) with additional structure. An isomorphism of trivialised sets is an isomorphism of stratified sets which preserves the additional structure.

We proceed by induction on the depth $d$ of the underlying stratified set $(V, \chi, \tau)$.

A trivialised set of depth 0 is a manifold. If $V^{\prime} \subset V$ are trivialised sets of depth 0 , $V^{\prime}$ is a restriction of $V$ if $V^{\prime}$ is a proper framed submanifold of $V$. If $V, V^{\prime}$ are trivialised sets of depth 0 , the product trivialised set of $V$ and $V^{\prime}$ is the manifold given by the product $V \times V^{\prime}$. An isomorphism between trivialised sets of depth 0 is a diffeomorphism $f: V \rightarrow V^{\prime}$.

Now assume that, for stratified sets of depth $<d$, we have defined

(a) the notion of a trivialised set (of depth $<d$ );

(b) the notion of restriction $V^{\prime} \subset V$ of trivialised sets;

(c) the product of a trivialised set $V$ (of depth $<d$ ) with a trivialised set $V^{\prime}$ of depth 0 ;

(d) the notion of isomorphism between trivialised sets (of depth $<d$ ).

Let $(V, \chi, \tau)$ be a stratified set of depth $d$ and assume, just for notational simplicity, that there is exactly one stratum $X \in \chi$ of codimension $d$.

$(V, \chi, \tau)$ is a trivialised set if there exists an open cone $D(X)$ on

$$
L(X)=L(X) \times\{1\} \subset D(X)
$$

and an isomorphism of (pairs of) stratified sets $h_{X}: T_{X}, \dot{V}_{X} \rightarrow X \times D(X), X \times L(X)$ such that:

(1) $V-X$ is a trivialised set (of depth $<d$ ) and $\left(T_{X}-X\right), \dot{V}_{X}$ are restrictions of $V-X$

(2) $L(X)$ is a trivialised set of depth $<d$ and $D(X)-\{0\}$ has the structure of a trivialised set induced by the canonical identification with $L(X) \times(0, \infty)$;

(3) $h_{X}$ restricts to an isomorphism of trivialised sets between $T_{X}-X, \dot{V}_{X}$ and $X \times(D(X)-\{0\}), X \times L(X)$ respectively, where $X \times(D(X)-\{0\})$ and $X \times$ $L(X)$ have a structure of trivialised sets by (c) above.

In order to complete the inductive definition, one needs to define the notions of restriction, product and isomorphism for trivialised sets of depth $d$. This is left to the reader. 
Most of the results of this paper will concern stratified sets, while trivialised sets will be used only as a tool. The main advantage of trivialised sets is that, if the link $L(X)$ is a boundary, then the stratum $X$ may be resolved by a blow-up; the same is not true for a general stratified set. However, the idea of a trivialised set is not without independent interest; for instance, it arises naturally in the following three situations:

(1) Sullivan's geometric description of connected $K$-theory at odd primes via join-like singularity $[13,2]$.

(2) In [4] it is proved that a transverse map $f: M \rightarrow X$ from a manifold to a transverse $\mathrm{CW}$-complex $X$ determines on $M$ a structure of a trivialised set (with additional properties) well-defined up to isomorphism.

(3) Levitt's and Akbulut-King's presentation of a $P L$ manifold as a (particular) trivialised set $[\mathbf{1}, \mathbf{1 8}]$.

The fundamental class of a stratified cycle. We are going to define a natural transformation of homology theories $F(A, B): H_{*}^{\chi}(A, B) \rightarrow H_{*}(A, B)$; for simplicity, let $B=\varnothing$ and $z=[f: V \rightarrow A] \in H_{n}^{\chi}(A)$. From excision and Lefschetz duality we have $H_{n}(V, S V) \cong \mathbf{Z}_{2} \oplus \cdots \oplus \mathbf{Z}_{2}$ (the summands corresponding to the connected components of $V-S V$ ).

Taking the sum of generators gives a class $[V] \in H_{n}(V, S V)$, called the fundamental class of $V$. From exactness and $\operatorname{dim} S V \leqslant n-2$, we have an isomorphism $\alpha_{*}$ : $H_{n}(V) \rightarrow H_{n}(V, S V)$, where $\alpha:(V, \varnothing) \rightarrow(V, S V)$ is inclusion. We define $F(A)(z)$ to be the class $f_{*} \alpha_{*}^{-1}[V]$, written also $\hat{z} . F(A, B)$ is similarly defined and, using the comparison theorem, it is easy to see that $F(-,-)$ is an equivalence of homology theories on finite $\mathrm{CW}$ pairs.

Let $V$ be a stratified set, $M$ a manifold and $f: V \rightarrow M$ a (continuous) map. Let $P \subset M$ be a closed submanifold. We say that $f$ is transverse to $P$ if, for each stratum $X$ of $V, f \mid X$ is transverse to $P$. If $x$ is a point of $M$ and $f$ is transverse to $x$, we say that $x$ is a regular value of $f$. From Sard's theorem we have that the set of regular values is dense in $M$.

The two propositions below are proved by standard (homological) arguments.

Let $M$ be a manifold of dimension $n$.

1.10 Proposition. Let $z=[f: V, \partial V \rightarrow M, \partial M] \in H_{n}^{\chi}(M, \partial M)$. Then $z=0$ if and only if, for each regular value $x \in M-\partial M, f^{-1}(x)$ consists of an even number of points (necessarily lying in $V-S V$ ).

Let $P$ be a closed $p$-dimensional submanifold of $M-\partial M$ with inclusion $i$ : $P \hookrightarrow M-\partial M$. Let $D: H_{q}(M, \partial M) \rightarrow H^{n-q}(M)$ be the Lefschetz duality isomorphism.

1.11 Proposition. Let $z=[f: V, \partial V \rightarrow M, \partial M] \in H_{q}^{\chi}(M, \partial M), p+q=n$, and assume that $f$ is transverse to $P$. Then the following equality holds in $H_{0}(P)$ : $\left(i^{*} D \hat{z}\right) \cap[P]=\left[f^{-1}(P) \rightarrow P\right]$.

In this paragraph we have considered the theory $H_{*}^{\chi}(-)$. However, it is clear that what has been said applies also to the theory of trivialised cycles $\bar{H}_{*}^{\chi}(-)$. 
2. A blow-up theorem. Let $V$ be a stratified $n$-set and $V^{\prime} \subset V$ a substratified set. Let $X$ be a stratum of the singularity $S V$; we say that $X$ is incident to $V^{\prime}$ if $X>Y$ for some stratum $Y$ of $V^{\prime}$.

2.1 Definition. $V^{\prime} \subset V$ is locally top-dimensional if $\operatorname{dim} V^{\prime}>\operatorname{dim} X$, for any stratum $X$ of $S V$ incident to $V^{\prime}$.

Figure 4 gives an example of a substratified set $V^{\prime}$ which is not locally topdimensional.

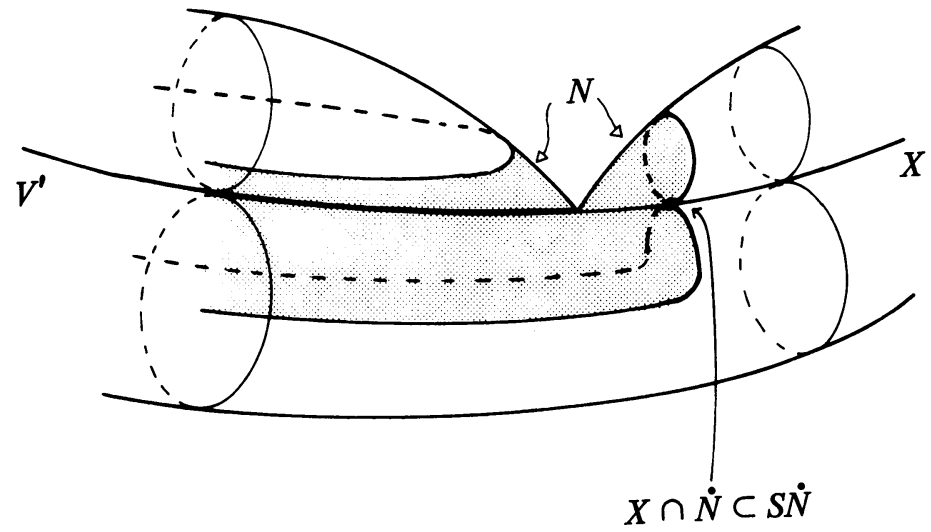

Figure 4

2.2 Remark. Let $N=T\left(V^{\prime}, V\right)$ and $X$ be a stratum of $S V$ incident to $V^{\prime}$. Then $X \cap \dot{N}$ is a stratum of $\dot{N}$ and $S \dot{N}=\cup_{X \in S V}(X \cap \dot{N})$. Moreover, $\operatorname{codim}_{\dot{N}}(X \cap \dot{N})$ $=\operatorname{codim}_{V} X$. Therefore, if we put $\beta=\beta\left(V^{\prime}, V\right)=\operatorname{codim}_{\dot{N}} S \dot{N}$ and $\gamma=\gamma\left(V^{\prime}, V\right)=$ $\operatorname{codim}_{V} V^{\prime}$, then $V^{\prime}$ is locally top-dimensional if and only if $\beta>\gamma$. Note that, in particular, if $\dot{N}$ is a manifold, then $V^{\prime}$ is locally top-dimensional.

Let $V$ be an unbounded stratified $n$-set, $V^{\prime}$ a substratified set of $V$ and $N=$ $T\left(V^{\prime}, V\right)$.

Let $\hat{V}$ be an unbounded stratified $n$-set, with $\hat{V}=P \cup \hat{N}$, where $P$ and $\hat{N}$ are bounded stratified $n$-sets such that $\dot{P}=\dot{\hat{N}}=P \cap \hat{N}$.

2.3 Definition. We say that the pair $(\hat{V}, \hat{N})$ is a blow-up of $(V, N)$ (or a blow-up of $V$ along $V^{\prime}$ ) if:

(a) $\operatorname{dim} S \hat{N} \leqslant \operatorname{dim} S N$;

(b) there exists a map $f:(\hat{V}, \hat{N}) \rightarrow(V, N)$ which restricts to an isomorphism $f$ : $P \rightarrow E_{V^{\prime}}$.

In order to simplify notation, we shall often identify $P$ with $E_{V^{\prime}}$ by means of $f$ (i.e. write $f$ as id on $P$ ).

2.4 REMARKs. (a) Note that the above definition does not require (as the definition of a blow-up usually does) that $\hat{N}$ deformation retracts onto $f^{-1}\left(V^{\prime}\right)$. However, this may always be achieved by "adding a collar": that is, using a collar of $\dot{N}$ in $N$, we may identify $\hat{V}$ with $E_{V^{\prime}} \cup_{\dot{N} \times\{1\}} \dot{N} \times[0,1] \cup_{\dot{N} \times\{0\}} \hat{N}$. Let $\pi: N \rightarrow V^{\prime}$ be a mapping cylinder projection for $N$. Then we define a blow-up map $g: \hat{V} \rightarrow V$ as follows: $g\left|E_{V^{\prime}}=\mathrm{id} ; g\right| \hat{N}=\pi f$ and $g \mid \dot{N} \times[0,1]$ is the quotient map $\dot{N} \times[0,1] \rightarrow N$. Then $g \simeq f$ and $\hat{N} \cup_{\dot{N} \times\{0\}} \dot{N} \times[0,1]$ deformation retracts onto $\hat{N}=g^{-1}\left(V^{\prime}\right)$. 
(b) When it is not necessary, we shall avoid mentioning the map $f$.

(c) Let $(\hat{V}, \hat{N})$ be a blow-up of $(V, N), N_{1} \subset \hat{N}$, and $\left(\hat{V}, \hat{N}_{1}\right)$ be a blow-up of $\left(\hat{V}, N_{1}\right)$; then the pair $(\overline{V-N} \cup \hat{N}, \hat{N})$, where

$$
\hat{N}=\left(\overline{\hat{N}-N_{1}}\right) \cup \hat{N}_{1},
$$

is a blow-up of $(V, N)$ which we call the composition of the blow-ups $\hat{V}$ and $\hat{V}$ (see Figure 5).

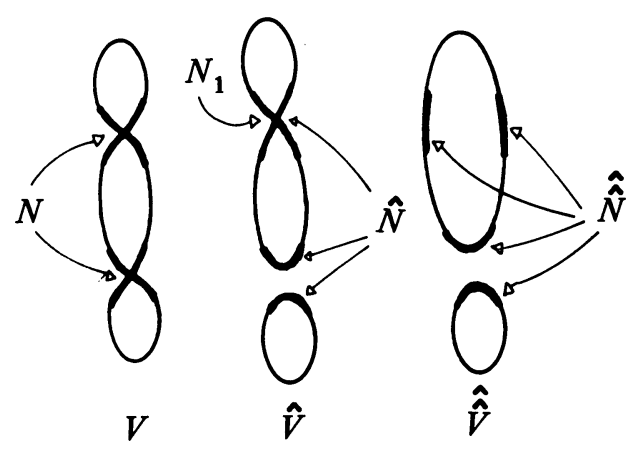

Figure 5

Let $\eta_{m}$ be the smooth unoriented bordism group of dimension $m$. For each $m \geqslant 0$, we fix a basis $\mathscr{B}_{m}=\left\{\left[M_{h}\right], h=1, \ldots, \operatorname{dim} \eta_{m}\right\}$ and characteristic classes $\sigma_{h}^{m} \in$ $H^{m}(-)$ (which are polynomials in the Stiefel-Whitney classes) such that the matrix of characteristic numbers $\left\{\sigma_{h}^{m}\left[M_{k}\right]\right\}$ is the unit matrix.

2.5 REMARK. In the following we shall be dealing only with spaces having the homotopy type of finite CW-complexes. Thus we may identify the groups $H_{n}(-)$, $H_{n}^{\chi}(-), \bar{H}_{n}^{\chi}(-)$.

Let $W$ be a compact unbounded stratified $n$-set and $m$ an integer such that $0 \leqslant m \leqslant(\operatorname{codim} S W)-2$. Choose a relative trivialised $(n-m)$-cycle $q_{h}: Q_{h}, \partial Q_{h}$ $\rightarrow E_{S W}, \dot{E}_{S W}$ Lefschetz dual to $\sigma_{h}^{m} \in H^{m}\left(E_{S W}\right)$. Since $T_{S W}$ deformation retracts onto $S W$ and $\operatorname{dim} S W<n-m-1=\operatorname{dim} \partial Q_{h}$, the cycle $q_{h} \mid: \partial Q_{h} \rightarrow \dot{E}_{S W}$ bounds in $H_{n-m-1}\left(T_{S W}\right)$. Thus one may choose a trivialised cycle $b_{h}: B_{h}, \partial B_{h} \rightarrow T_{S W}, \dot{T}_{S W}$ such that $\partial B_{h}=\partial Q_{h}$ and $b_{h}\left|\partial B_{h}=q_{h}\right| \partial Q_{h}$; form the union $A_{h}=B_{h} \cup_{\partial} Q_{h}, a_{h}: A_{h} \rightarrow W$, $a_{h}=q_{h} \cup b_{h} ;\left(A_{h}, a_{h}\right)$ is a closed trivialised $(n-m)$-cycle in $W$.

2.6 Proposition. The homology class of $\left(A_{h}, a_{h}\right)$ does not depend on the choice of the representatives $\left(Q_{h}, q_{h}\right)$ and $\left(B_{h}, b_{h}\right)$.

Proof. Let $r: R \rightarrow E_{S W}$ be a homology between $\left(Q_{h}, q_{h}\right)$ and another representative $\left(Q_{h}^{\prime}, q_{h}^{\prime}\right) ; r$ restricts to a homology $r \mid: R_{\partial} \rightarrow E_{S W}$ between $\partial Q_{h}$ and $\partial Q_{h}^{\prime}$. Then the union $U=B_{h} \cup R_{\mathrm{\partial}} \cup B_{h}^{\prime}$ is a closed $(n-m)$-cycle in $T_{S W}$ and the map $u$ : $U \rightarrow T_{S W}$ is given by $u=b_{h} \cup r \mid \cup b_{h}^{\prime}$ (see Figure 6); since, as before, $\operatorname{dim} U>$ $\operatorname{dim} S W$, this cycle bounds in $H_{n-m}\left(T_{S W}\right)$, hence there exists a cycle $p: P \rightarrow T_{S W}$ such that $\partial P=U$ and $p \mid U=u$. Finally form the cycle $Z=R \cup_{R_{\partial}} P$, whose boundary is $A_{h}+A_{h}^{\prime}$. There is a map $z: Z \rightarrow W$ given by $z=r \cup p$ and such that $z\left|A_{h}=a_{h}, z\right| A_{h}^{\prime}=a_{h}^{\prime}$. Therefore $(Z, z)$ is a homology between $\left(A_{h}, a_{h}\right)$ and $\left(A_{h}^{\prime}, a_{h}^{\prime}\right)$. 


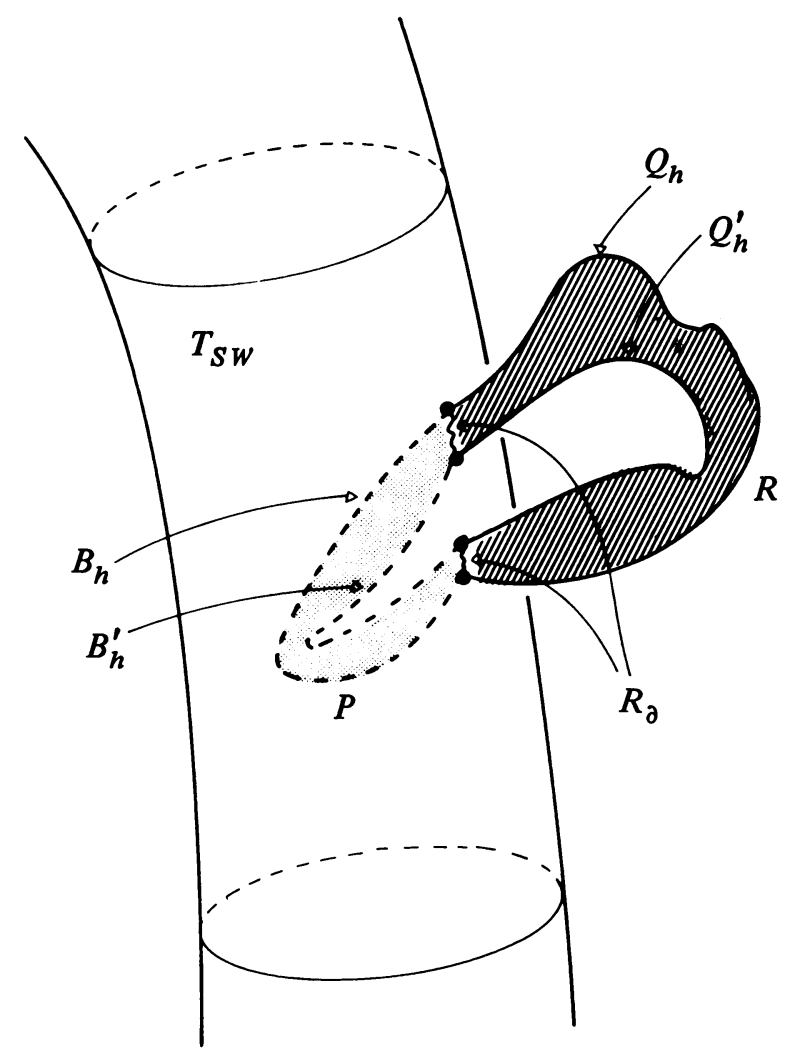

FiguRE 6

Thus the above construction gives a well-defined homology class $\mu_{n-m}^{h}(W) \in$ $H_{n-m}(W)$.

2.7 Remarks. (a) If $S W=\varnothing$, then $\mu_{n-m}^{h}(W)$ coincides with the Poincare dual of $\sigma_{h}^{m} \in H^{m}(W)$.

(b) Let $W$ be a relative stratified $n$-cycle and $m \geqslant 0$ be an integer such that $m \leqslant \operatorname{codim} S W-2$ and $m \leqslant \operatorname{codim} S(\partial W)-2$; then, by relativising the above construction in an obvious way, one obtains well-defined relative classes $\mu_{n-m}^{h}(W, \partial W)$.

In the following, let $V$ be a compact unbounded stratified $n$-set, $V^{\prime} \subset V$ a locally top-dimensional substratified set, $N=T\left(V^{\prime}, V\right), \beta=\operatorname{codim}_{\dot{N}} S \dot{N}$. Applying the above construction to $W=\dot{N}$, for each $m=0, \ldots, \beta-2$ and each $h=1, \ldots, \operatorname{dim} \eta_{m}$, we have the homology class $\mu_{n-m-1}^{h}(\dot{N})$. Recall that $N$ is the mapping cylinder of $\dot{\pi}$ : $\dot{N} \rightarrow V^{\prime}$. Then, associated to $V^{\prime}$, we define classes $\vartheta_{n-m-1}^{h}\left(V^{\prime}\right)=\dot{\pi}_{*} \mu_{n-m-1}^{h}(\dot{N}) \in$ $H_{n-m-1}\left(V^{\prime}\right)$.

In this section we shall prove that the classes $\vartheta_{i}^{h}\left(V^{\prime}\right)$ are the only obstructions to the existence of a blow-up $(\hat{V}, \hat{N})$ of $V$ along $V^{\prime}$ which increases the codimension of the singularity of $V$ up to a certain stage. 
2.8 LEMMA. Let $V$ be a compact unbounded trivialised n-set and $V^{\prime} \subset V$ a locally top-dimensional substratified set with $\operatorname{codim}_{V} V^{\prime}=\gamma$. Suppose that, for each top-dimensional stratum $X$ in $V^{\prime},[L(X, V)]=0$ in $\eta_{\gamma-1}$. Then there exists a blow-up $f$ : $(\hat{V}, \hat{N}) \rightarrow(V, N)$ of $V$ along $V^{\prime}$ such that $\operatorname{codim} S \hat{N}>\gamma$.

Proof. For each top-dimensional stratum $X$ in $V^{\prime}$, choose a manifold $M(X)$ such that $\partial M(X)=L(X)$. Let $\hat{V}, \hat{N}$ be the stratified set obtained from $V, N$ by replacing $\bar{X} \underset{\sim}{\times} C(X)$ with $\bar{X} \underset{\sim}{\times} M(X)$ for each $X$. Choose a map $f_{X}: M(X) \rightarrow C(X)=c L(X)$ which identifies an open collar of $L(X)$ in $M(X)$ with the punctured cone $c L(X)-$ $\{0\}$ and maps the rest of $M(X)$ to 0 . Then a blow-up $f:(\hat{V}, \hat{N}) \rightarrow(V, N)$ of $V$ along $V^{\prime}$ is constructed using $f_{X}$ on each fibre of $\bar{X} \underset{\sim}{\times} M(X)$ and the identity elsewhere.

Note that $\hat{V}$ depends on the choice of the manifolds $M(X)$.

2.9 LEMMA. Let $V$ be a compact unbounded trivialised $n$-set and $V^{\prime} \subset V$ a locally top-dimensional substratified set with $\operatorname{codim}_{V} V^{\prime}=\gamma$. Then the following statements are equivalent:

(a) $\vartheta_{n-\gamma}^{h}\left(V^{\prime}\right)=0$, for each $h=1, \ldots, \operatorname{dim} \eta_{\gamma-1}$;

(b) there exists a blow-up $(\hat{V}, \hat{N})$ of $V$ along $V^{\prime}$ with $\operatorname{codim} S \hat{N}>\gamma$.

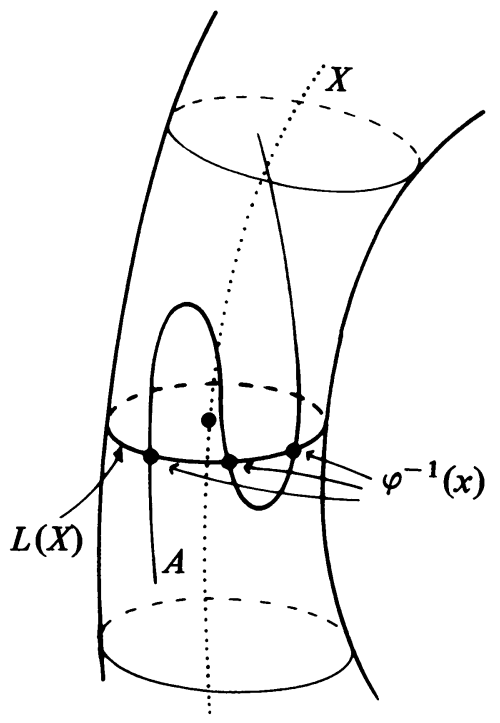

FIGURE 7

Proof. (a) $\Rightarrow$ (b). Let $X$ be a top-dimensional stratum of $V^{\prime}$. Then $L(X)=L(X, V)$ is necessarily a manifold because $V^{\prime}$ is locally top-dimensional. By the previous lemma, we only need to prove that $L(X)$ is a boundary. Fix $h$; a representative of $\vartheta_{n-\gamma}\left(V^{\prime}\right)=\vartheta_{n-\gamma}^{h}\left(V^{\prime}\right)$ is, by definition,

$$
\varphi: A \stackrel{a}{\rightarrow} \dot{N} \stackrel{\dot{n}}{\rightarrow} V^{\prime},
$$

where $(A, a)=(Q \cup B, q \cup b)$ and $(Q, q)$ is a Lefschetz dual of $\sigma_{h}^{\gamma-1} \in H^{\gamma-1}\left(E_{S \dot{N}}\right)$. 
From excision and homotopy it follows easily that $[A, \varphi]=0$ if and only if $[Q, \dot{\pi} q]=0$ in $E\left(S V^{\prime}, V^{\prime}\right)$. Let $x \in \underline{X}-\partial \underline{X}$ be a regular value for $\dot{\pi} q$; then $[A, \varphi]=0$ implies that $\varphi^{-1}(x)$ consists of an even number of points (by Proposition 1.10).

By the definition of a trivialised set $\dot{\pi}: V_{X} \rightarrow \underline{X}$ may be identified with projection $\underline{X} \times L(X) \rightarrow \underline{X}$; in particular, $x$ is certainly a regular value for the restriction of $\dot{\pi}$ and its inverse image is $\dot{\pi}^{-1}(x) \cong L(X)$. This implies that $q: Q \rightarrow E_{S \dot{N}}$ meets $\dot{\pi}^{-1}(x)$ transversely in a finite number of points. At the cohomology level this corresponds to restricting the class $\sigma_{h}^{\gamma-1}$ to $\dot{\pi}^{-1}(X) \cong L(X)$ (see Proposition 1.11). Since $\sigma_{h}^{\gamma-1}$ is a characteristic class and $\dot{\pi}^{-1}(x)$ is framed in $E_{S \dot{N}}$, the Poincaré dual of $\sigma_{h}^{\gamma-1} \mid \dot{\pi}^{-}(x)$ is a set of points whose sum $(\bmod 2)$ is exactly the characteristic number $\sigma_{h}^{\gamma-1}[L(X)]$ (see Figure 7). Since this is equal to the number of points in $\varphi^{-1}(x)$, we have proved that $\sigma_{h}^{\gamma-1}[L(X)]=0$.

As this happens for every $h=1, \ldots, \operatorname{dim} \eta_{\gamma-1}$, we deduce that $L(X)$ is a boundary.

Note that, if $\gamma=1$, we have just one class $\vartheta_{n-1}\left(V^{\prime}\right)$ which is represented by $\dot{\pi}$ : $\dot{N} \rightarrow V^{\prime}$. Thus $L(X) \cong \dot{\pi}^{-1}(x)$ consists of an even number of points.

(b) $\Rightarrow$ (a). Let $f:(\hat{V}, \hat{N}) \rightarrow(V, N)$ be a blow-up of $V$ along $V^{\prime}$, such that $\operatorname{codim} S \hat{N}$ $>\gamma$. As also codim $S \dot{\hat{N}}=\operatorname{codim} S \dot{N}=\beta>\gamma$, by Remark 2.7(b) we can define classes $\mu_{n-\gamma+1}^{h}(\hat{N}, \dot{N})$. We fix a particular index $h$ and, for simplicity, we drop it from the notation. Consider the following diagram:

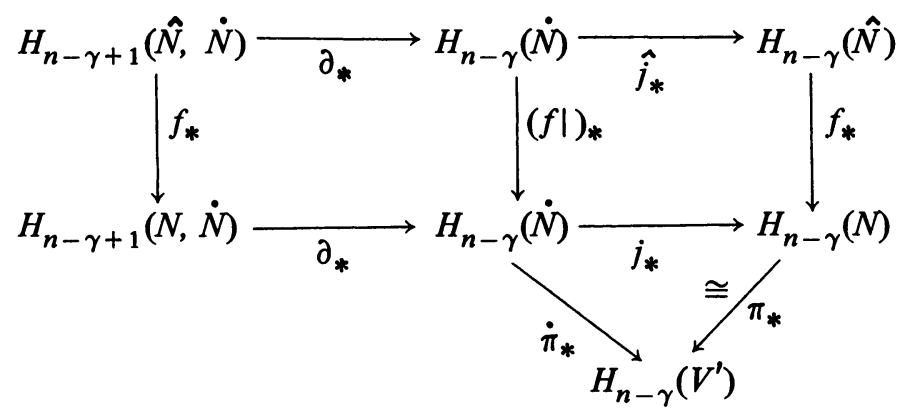

By construction, we have $\partial_{*} \mu_{n-\gamma+1}(\hat{N}, \dot{N})=\mu_{n-\gamma}(\dot{N})$ and therefore $\hat{j}_{*} \mu_{n-\gamma}(\dot{N})$ $=0$. Thus

$$
\vartheta_{n-\gamma}\left(V^{\prime}\right)=\dot{\pi}_{*} \mu_{n-\gamma}(\dot{N})=\pi_{*} j_{*} \mu_{n-\gamma}(\dot{N})=\pi_{*} f_{*} \hat{j}_{*} \mu_{n-\gamma}(\dot{N})=0
$$

as required.

The idea is now to go on inductively, that is to prove that the classes $\vartheta_{n-\gamma-1}^{h}$ vanish if and only if there exists a blow-up $(\hat{V}, \hat{N})$ of $V$ along $V^{\prime}$ with codim $S \hat{N}>\gamma$ +1 . Of course we must assume $\beta>\gamma+1$, otherwise the classes $\vartheta_{n-\gamma-1}^{h}$ are not defined. More precisely, we wish to prove the following

2.10 TheOREM. Let $V$ be a compact unbounded trivialised $n$-set, $V^{\prime} \subset V$ a locally top-dimensional substratified set, $N=T\left(V^{\prime}, V\right), \beta=\operatorname{codim}_{\dot{N}} S \dot{N}, \gamma=\operatorname{codim}_{V} V^{\prime}$. Let $k$ be an integer such that $k<\beta-\gamma+1$ (so that the classes $\mu_{n-m-1}^{h}(\dot{N})$ are defined, for each $m \leqslant \gamma+k-2 \leqslant \beta-2)$. Then the following statements are equivalent:

(a) all the classes $\vartheta_{n-m-1}^{h}\left(V^{\prime}\right)$ vanish, for each $m=\gamma-1, \ldots, \gamma+k-2$ and for each $h=1, \ldots, \operatorname{dim} \eta_{m}$;

(b) there exists a blow-up $(\hat{V}, \hat{N})$ of $V$ along $V^{\prime}$ such that $\operatorname{codim} S \hat{N} \geqslant \gamma+k$. 
Note that, if $\beta=1$, this theorem is vacuous.

The lemma we have just proved is the first stage in proving the theorem: however we cannot just iterate the lemma since, once one has constructed the blow-up $(\hat{V}, \hat{N})$ of $(V, N)$ such that codim $S \hat{N}>\gamma$, it is no longer true that, for a top-dimensional stratum $X$ in $\hat{N}, L(X)$ is necessarily a boundary. Instead, the proof will go through the following stages.

(a) Let $X$ be a top-dimensional stratum in $\hat{N}$ such that $\bar{X}$ is a cycle and $L(X, \hat{V})=M_{h}$, with $\left[M_{h}\right] \in \mathscr{B}_{\gamma}$. Then we shall prove that the vanishing of the corresponding obstruction $\vartheta_{n-\gamma-1}^{h}\left(V^{\prime}\right)$ implies $f_{*}([\bar{X}])=0$ in $H_{n-\gamma-1}\left(V^{\prime}\right)$.

(b) From the vanishing of $f_{*}([\bar{X}])$, for each top-dimensional stratum $X$ in $\hat{N}$, we shall construct a new blow-up of $(V, N)$ with singularity in codimension $>\gamma+1$.

(c) We iterate this procedure.

Of course, $\bar{X}$ may well fail to be a cycle, because there may exist strata $Y<X$ with $\cdot \operatorname{dim} Y=\operatorname{dim} X-1$. So, first of all, we shall prove the following

2.11 LemMA. Let $V$ be a compact unbounded trivialised n-set and $V^{\prime}$ a locally top-dimensional substratified set such that $\operatorname{dim} V^{\prime}=n-\gamma-1$, and let $N=T\left(V^{\prime}, V\right)$. Then there exists a blow-up $(\tilde{V}, \tilde{N})$ of $V$ along $V^{\prime}$ such that, if $\tilde{X}$ is any top-dimensional stratum of $S \tilde{N}$, then:

(a) $[\underline{L}(\tilde{X}, \tilde{V})] \in \mathscr{B}_{\gamma}$;

(b) $\overline{\tilde{X}}$ is a trivialised $(n-\gamma-1)$-cycle;

(c) if $\tilde{Y}$ is any other top-dimensional stratum of $S \tilde{N}$ and $\tilde{X} \neq \tilde{Y}$, then $[L(\tilde{X}, \tilde{V})] \neq$ $[L(\tilde{Y}, \tilde{V})]$.

Proof. Let $X$ be a top-dimensional stratum of $V^{\prime}$. Then $L(X)=L(X, V)$ is a $\gamma$-dimensional manifold, bordant to a sum $M_{1}+\cdots+M_{r}$, where $\left[M_{i}\right] \in \Re_{\gamma}$. We wish to "replace" $X$ by $r$ copies $X_{1}, \ldots, X_{r}$ of $X$, with $L\left(X_{i}\right)=M_{i}$. The construction is essentially the same as in Lemma 2.8 (where $\left.\left[M_{1}\right]=\cdots=\left[M_{r}\right]=0\right)$. Let $W^{\prime}$ be a bordism between $L(X)$ and $M_{1}+\cdots+M_{r}$ and define $W=W^{\prime} \cup\left(c_{1} M_{1}\right.$ $\left.+\cdots+c_{r} M_{r}\right)$, where $c_{i} M_{i}$ is the cone over $M_{i}$ with vertex $c_{i}$ and the union is taken along $M_{1}+\cdots+M_{r}$. Choose a map $f_{W}: W \rightarrow C(X)=c L(X)$ which identifies an open collar of $L(X)$ in $W$ with the punctured cone $C(X)-\{0\}$ and takes the rest of $W$ to the vertex. Then a blow-up $\left(V^{*}, N^{*}\right)$ of $(V, N)$ is constructed by replacing $\bar{X} \underset{\sim}{\times} C(X)$ with $\bar{X} \underset{\sim}{\times} W$; the map $f:\left(V^{*}, N^{*}\right) \rightarrow(V, N)$ is defined using the map $f_{W}$ on each fibre of $\bar{X} \underset{\sim}{\times} W$ and the identity elsewhere.

Clearly, if we do the above construction on each top-dimensional stratum $X$ in $V^{\prime}$, the blow-up $\left(V^{*}, N^{*}\right)$ satisfies property (a); thus (see Remark 2.4(c)) we can assume that the stratified $n$-set $V$ already satisfies (a).

Now, let $Y$ be a stratum of $V^{\prime}$ such that $\operatorname{dim} Y=n-\gamma-2$ and $X_{1}, \ldots, X_{r}$ be the top-dimensional strata of $V^{\prime}$ such that $Y<X_{1}, \ldots, Y<X_{r}$. Let $W=L(Y, V)$; $W$ is a trivialised $(\gamma+1)$-set with isolated singularities $S W=\left\{Y_{1}, \ldots, Y_{r}\right\}$ and, if $Y_{i}=X_{i}$ $\cap W$ is a singular point of $W$, we have $L\left(Y_{i}, W\right)=L\left(X_{i}, V\right) \in \mathscr{B}_{\gamma}$. We wish to "replace" $c W$ by a trivialised set $W^{\prime}$ such that $S W^{\prime}=\{*\}+\{1$-dimensional manifolds with boundary $\} E(S W, W)$ is a $(\gamma+1)$-manifold, whose boundary is $L\left(Y_{1}, W\right)+\cdots+L\left(Y_{r}, W\right)$; as $L\left(Y_{i}, W\right)=L\left(X_{i}, V\right) \in \mathscr{B}_{\gamma}$, to each $X_{i}$ there must 


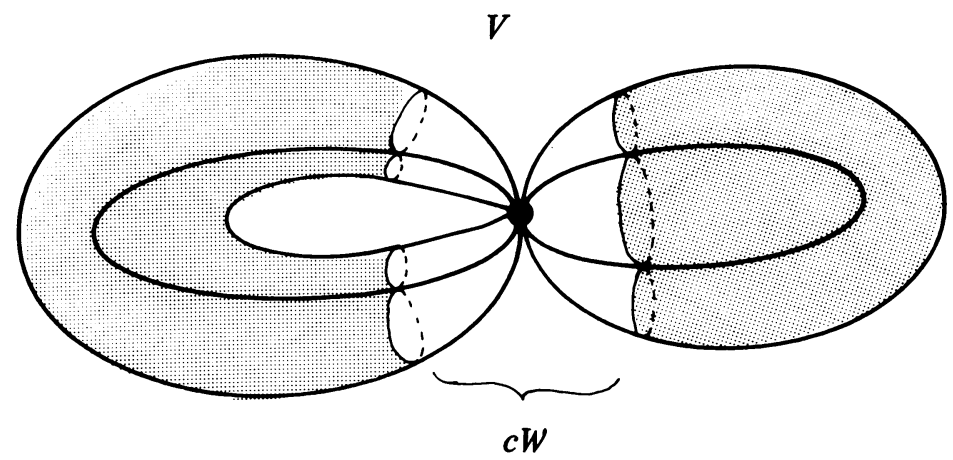

FigURE 8

correspond an $X_{j}$ such that $L\left(X_{i}\right)$ is bordant to $L\left(X_{j}\right)$ (see Figure 8); w.l.o.g. we may assume $L\left(X_{i}\right)=L\left(X_{j}\right)=L$. Then $W^{\prime}$ is obtained as follows: embed $W$ as $W \times\{0\}$ in $W \times[0,1 / 2]$ and, for each pair $X_{i}, X_{j}$ as above, attach a "handle" [1,2] $\times c L$ by identifying $\{1\} \times c L$ with $Y_{i} \times c L$ and $\{2\} \times c L$ with $Y_{j} \times c L$. We obtain a trivialised $(\gamma+1)$-cycle $Q$ such that $\partial Q=W \times\{1 / 2\}+M$, where $M$ is a manifold (see Figure 9). Finally define $W^{\prime}=Q \cup_{M} c M$. $W^{\prime}$ is a trivialised $(\gamma+2)$-set with the required properties, $\partial W^{\prime}=W$ and, in the usual way, we may construct a map $f_{W}$ : $W^{\prime} \rightarrow c W$ such that $f_{W} \mid W=1_{W}$.

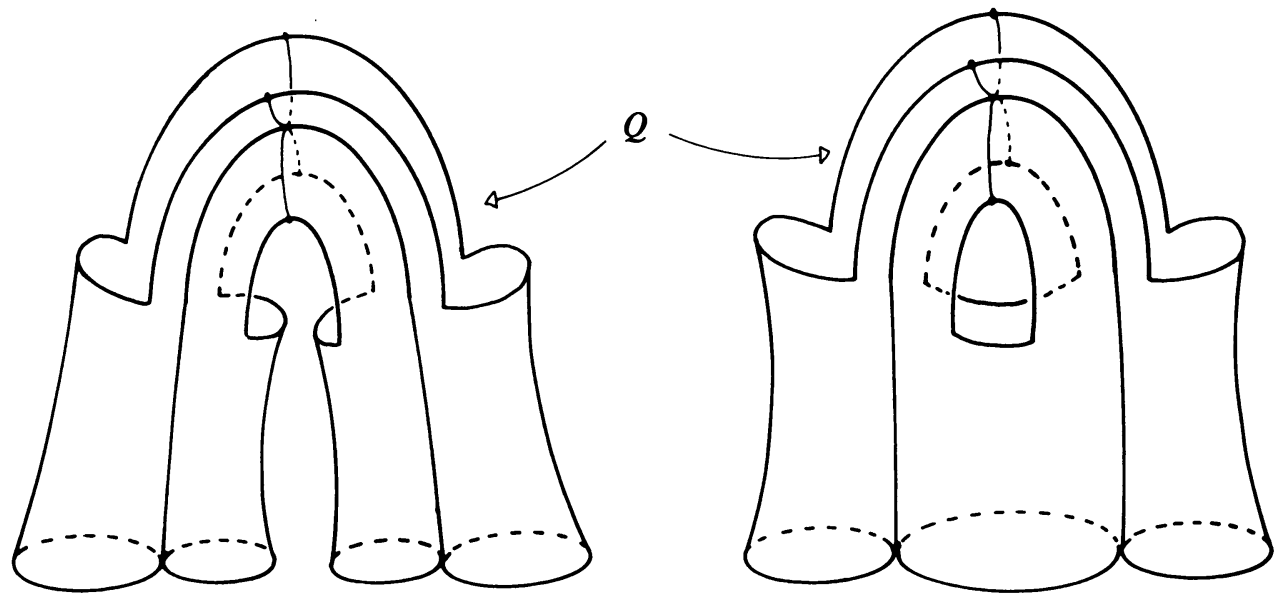

Figure 9

Now let $(\tilde{V}, \tilde{N})$ be the trivialised set obtained from $(V, N)$ by replacing each fibre $\bar{Y} \times \underset{\sim}{\times} w$ with $\bar{Y} \underset{\sim}{\times} W^{\prime}$, for each $(n-\gamma-2)$-dimensional stratum $Y$ of $V^{\prime}$ (such that $Y<X_{i}$ for more than two $(n-\gamma-1)$-dimensional strata $X_{i}$ of $\left.V^{\prime}\right)$. There is an obvious map $f:(\tilde{V}, \tilde{N}) \rightarrow(V, N)$ constructed from the maps $f_{W}$ (see Figures 8 and 10). $(\tilde{V}, \tilde{N})$ is the required blow-up: in fact it satisfies properties (a) and (b) and, in order to satisfy property (c), it is enough to "unite" any two $(n-\gamma-1)$-dimensional strata $\tilde{X}$ and $\tilde{Y}$ such that $[L(\tilde{X}, \tilde{V})]=[L(\tilde{Y}, \tilde{V})]$. 


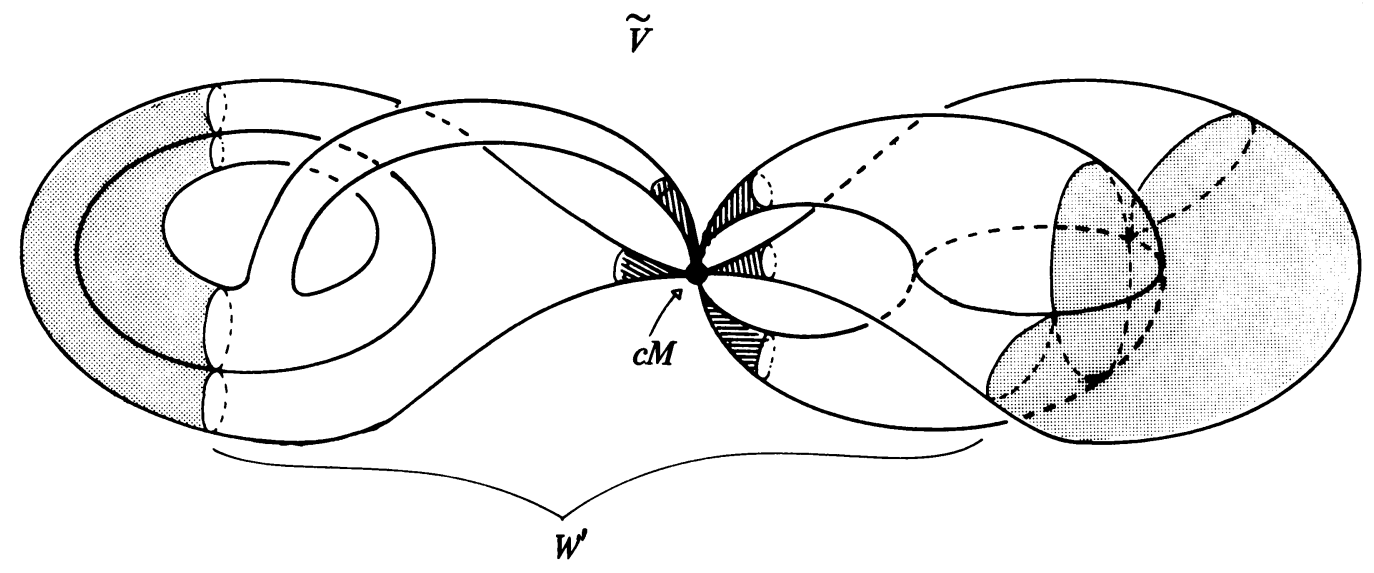

FIGURE 10

2.12 LeMma. Let $V$ be a compact unbounded trivialised n-set, $V^{\prime} \subset V$ a locally top-dimensional substratified set and $f:(\tilde{V}, \tilde{N}) \rightarrow(V, N)$ a blow-up of $V$ along $V^{\prime}$ such that:

(a) for each top-dimensional stratum $\tilde{X}$ in $S \tilde{N}, \overline{\tilde{X}}$ is a trivialised $k$-cycle;

(b) $f_{*}[\bar{X}]=0$ in $H_{k}\left(V^{\prime}\right)$ (or, equivalently, in $H_{k}(N)$ ).

Then there exists another blow-up $g:(\hat{V}, \hat{N}) \rightarrow(V, N)$ of $V$ along $V^{\prime}$ such that codim $S \hat{N}>\operatorname{codim} S \tilde{N}$. (Note the special case $f=\mathrm{id}$.)

Proof. Let $\tilde{X}_{1}, \ldots, \tilde{X}_{q}$ be the top-dimensional strata of $S \tilde{N}$. For each $i=1, \ldots, q$, let $\left(D_{i}, d_{i}\right)$ be a homology to zero of $f_{*}\left[\overline{\tilde{X}}_{i}\right]$, that is $D_{i}$ is a trivialised $(k+1)$-cycle, $\partial D_{i}=\overline{\tilde{X}}_{i}$ and $d_{i}: D_{i} \rightarrow N$ is a map such that $d_{i} \mid \partial D_{i}=f$. Let

$$
U=\sum_{i=1, \ldots, q} D_{i} \underset{\sim}{\times} M_{i} ; \quad \partial U=\sum_{i=1, \ldots, q} \overline{\tilde{X}}_{i} \underset{\sim}{\times} M_{i}=\sum_{i=1, \ldots, q} \overline{\tilde{X}}_{i} \underset{\sim}{\times} L\left(\tilde{X}_{i}, \tilde{V}\right) .
$$

Define

$$
R=\bigcap_{i=1, \ldots, q} \overline{\tilde{V}-\left(\overline{\tilde{X}}_{i} \underset{\sim}{\times} C\left(\tilde{X}_{i}\right)\right)}, \quad \hat{V}=R \underset{\partial \widetilde{\partial U}}{\cup} U
$$

and

$$
\hat{N}=(R \cap \tilde{N}) \underset{\partial U}{\cup} U
$$

There is a natural blow-up map $g:(\hat{V}, \hat{N}) \rightarrow(V, N)$ obtained from the composition of the projections $D_{i} \times M_{i} \rightarrow D_{i}$ and the maps $d_{i}$ (see Figure 11). Moreover, $\operatorname{dim} S \hat{N} \leqslant \max _{i} \operatorname{dim} S D_{i} \leqslant k-1$, so that $(\hat{V}, \hat{N})$ is the required blow-up.

PROOF OF THEOREM 2.10. The proof of (b) $\Rightarrow$ (a) goes exactly as in Lemma 2.9.

In order to prove $(\mathrm{a}) \Rightarrow(\mathrm{b})$, let $\left(V_{1}, N_{1}\right)$ be the blow-up of $(V, N)$ constructed as in Lemma 2.9 using the vanishing of the classes $\boldsymbol{\vartheta}_{n-\gamma}^{h}\left(V^{\prime}\right)$. If $\beta>\gamma+1$ (otherwise the theorem is already proved), let $(\tilde{V}, \tilde{N})$ be a blow-up of $\left(V_{1}, N_{1}\right)$ satisfying properties (a), (b), (c) of Lemma 2.11. 

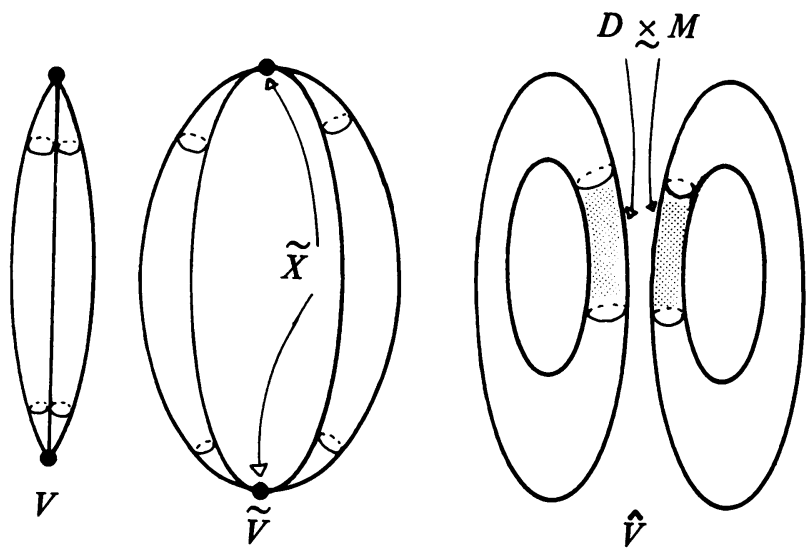

Figure 11

Let $\tilde{X}_{1}, \ldots, \tilde{X}_{q}$ be the top-dimensional strata of $S \tilde{N}$. $\overline{\tilde{X}}_{i}$ is a trivialised $(n-\gamma-1)$ cycle, and, by the previous lemma, we only need to prove that $f_{*}\left[\overline{\tilde{X}}_{i}\right]=0$ in $H_{n-\gamma-1}\left(V^{\prime}\right)$, where $f:(\tilde{V}, \tilde{N}) \rightarrow(V, N)$ is the blow-up map. To do this, we shall use the vanishing of the obstructions $\vartheta_{n-\gamma-1}^{h}\left(V^{\prime}\right)$. After reordering the strata $\tilde{X}_{1}, \ldots, \tilde{X}_{q}$, we may suppose that $\left[L\left(\tilde{X}_{h}, \tilde{V}\right)\right]=\left[M_{h}\right]$. Let $\tilde{V}^{\prime}$ be the closure of $\left\{\tilde{X}_{1}, \ldots, \tilde{X}_{q}\right\}$ and $Q=T\left(\tilde{V}^{\prime}, \tilde{V}\right)$. We may assume $Q \subset \tilde{N}-\tilde{N}^{2}$; let $\tilde{\pi}: Q \rightarrow \tilde{V}^{\prime}$ be the mapping cylinder retraction and $\tilde{\pi}=\tilde{\pi} \mid \dot{Q}$. As $\hat{N}^{2}=\dot{N}$ and $\beta>\gamma+1$, we have well-defined classes $\mu_{n-\gamma-1}^{h}(\dot{Q})$ and $\vartheta_{n-\gamma-1}^{h}\left(\tilde{V}^{\prime}\right)=\tilde{\pi}_{*} \mu_{n-\gamma-1}^{h}(\dot{Q})$. In $H_{n-\gamma-1}\left(\tilde{V}^{\prime}\right)$ the class $\vartheta_{n-\gamma-1}^{h}\left(\tilde{V}^{\prime}\right)$ will be equal to a linear combination (with $\mathbf{Z}_{2}$ coefficients)

$$
\vartheta_{n-\gamma-1}^{h}\left(\tilde{V}^{\prime}\right)=\sum_{i=1, \ldots, q} k_{i}\left[\bar{X}_{i}\right]
$$

where $\left[\overline{\tilde{X}}_{i}\right]$ is the homology class of $\overline{\tilde{X}}_{i} \subset \tilde{V}^{\prime}$. But, using the same kind of arguments as in Lemma 2.9 , one proves that $k_{i}$ is exactly the characteristic number

Then

$$
\sigma_{h}^{\gamma}\left[L\left(\tilde{X}_{i}, \tilde{V}\right)\right]=\sigma_{h}^{\gamma}\left[M_{i}\right]= \begin{cases}1 & \text { if } i=h, \\ 0 & \text { if } i \neq h .\end{cases}
$$

$$
\vartheta_{n-\gamma-1}^{h}\left(\tilde{V}^{\prime}\right)= \begin{cases}0 & \text { if } h>q, \\ {\left[\overline{\tilde{X}}_{h}\right]} & \text { if } h \leqslant q .\end{cases}
$$

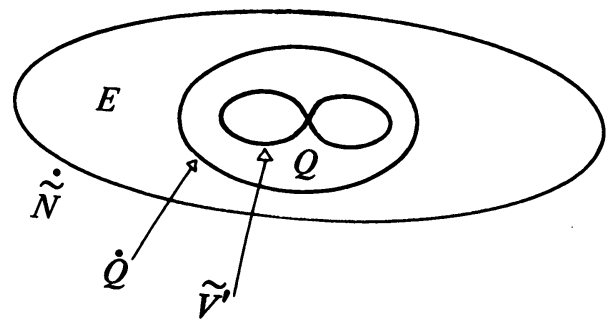

Figure 12 
Then we only need to prove that $f_{*} \vartheta_{n-\gamma-1}^{h}\left(\tilde{V}^{\prime}\right)=0$. Let $E=E\left(\tilde{V}^{\prime}, \tilde{N}\right) ; E$ is a trivialised $n$-set bounded by $\dot{E}=\hat{N}^{2}+\dot{Q}$ (see Figure 12). Furthermore, by construction, codim $S E$ and codim $S \dot{E}$ are both greater than $\gamma$. Therefore, there are well defined classes $\mu_{n-\gamma}^{h}(E, \dot{E})$ and

$$
\partial_{*} \mu_{n-\gamma}^{h}(E, \dot{E})=\mu_{n-\gamma-1}^{h}\left(\dot{N}^{2}\right)+\mu_{n-\gamma-1}^{h}(\dot{Q}) \text {. }
$$

Thus

$$
f_{*} \tilde{j}_{*} \mu_{n-\gamma-1}^{h}(\stackrel{2}{N})+f_{*} \tilde{j}_{*} \mu_{n-\gamma-1}^{h}(\dot{Q})=0
$$

(see the following diagram). But

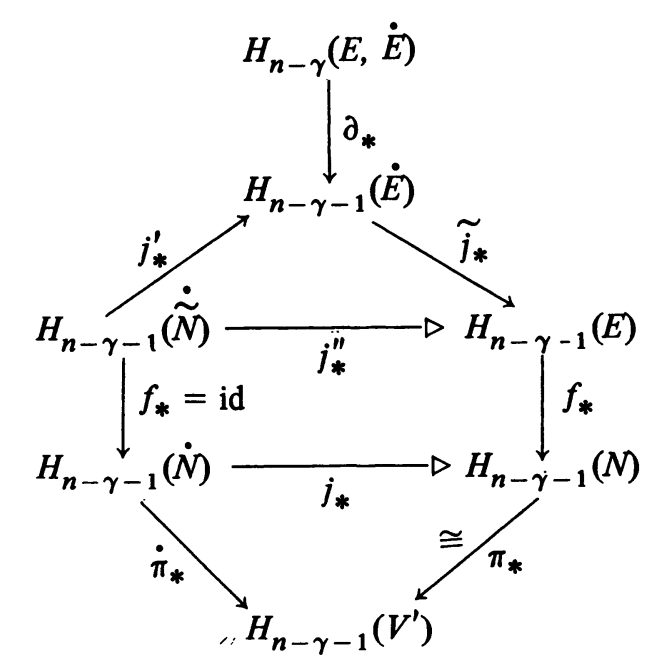

$$
\begin{aligned}
f_{*} \tilde{j}_{*} \mu_{n-\gamma-1}^{h}(\dot{N}) & =f_{*} j_{*}^{\prime \prime} \mu_{n-\gamma-1}^{h}(\stackrel{2}{N}) \\
& =j_{*} f_{*} \mu_{n-\gamma-1}^{h}(\stackrel{2}{N})=j_{*} \mu_{n-\gamma-1}^{h}(\dot{N}) \\
& =\pi_{*}^{-1} \dot{\pi}_{*} \mu_{n-\gamma-1}^{h}(\dot{N})=\pi_{*}^{-1} \vartheta_{n-\gamma-1}^{h}\left(V^{\prime}\right)=0,
\end{aligned}
$$

by our hypothesis. Thus, in (*), we have

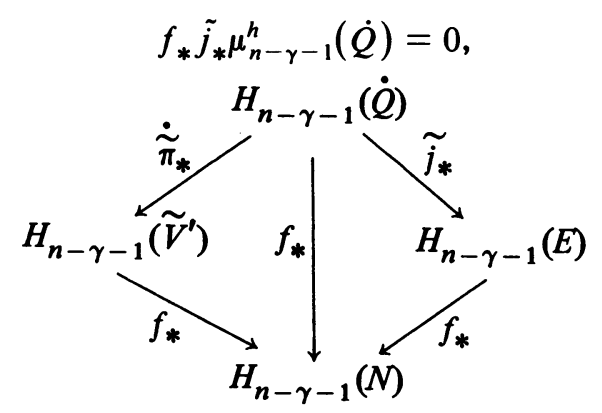

and

$$
\begin{aligned}
f_{*} \vartheta_{n-\gamma-1}^{h}\left(\tilde{V}^{\prime}\right) & =f_{*} \tilde{\pi}_{*} \mu_{n-\gamma-1}^{h}(\dot{Q}) \\
& =f_{*} \tilde{j}_{*} \mu_{n-\gamma-1}^{h}(\dot{Q})=0
\end{aligned}
$$

as we wanted. 
Now we can use Lemma 2.12 to construct a blow-up $\left(V_{2}, N_{2}\right)$ of $V$ along $V^{\prime}$ such that $\operatorname{codim} S N_{2}>\gamma+1$. If $\beta=\gamma+2$, the theorem is proved; if $\beta>\gamma+2$, the same procedure may be carried on without any new difficulty until we reach the stage where singularities are in codimension $\geqslant \gamma+k$. So the theorem is proved.

Note that the vanishing of the obstructions has been used only in Lemma 2.9 and in this final proof, while Lemmas 2.8, 2.11 and 2.12 are only geometric constructions on the trivialised set $V$, which do not involve the vanishing of the classes $\vartheta_{n-m-i}^{h}\left(V^{\prime}\right)$. These lemmas use in an essential way the fact that $V$ is trivialised; however, we wish to extend 2.10 to the more natural case where $V$ is not necessarily trivialised.

2.13 THEOREM. Let $V$ be a compact unbounded stratified $n$-set and $V^{\prime}, N, \beta, \gamma, k$ be as in 2.10. Then the following statements are equivalent:

(a) $\vartheta_{n-m-1}^{h}\left(V^{\prime}\right)=0$ for each $m=0, \gamma-1, \ldots, \gamma+k-2$ and for each $h=$ $1, \ldots, \operatorname{dim} \eta_{m}$

(b) there exists a blow-up $(\hat{V}, \hat{N})$ of $V$ along $V^{\prime}$ such that $\operatorname{codim} S \hat{N} \geqslant \gamma+k$.

Proof. We consider a variant $H_{n}^{\chi}(-; \beta)$ of the theory $H_{n}^{\chi}(-) . H_{n}^{\chi}(-; \beta)$ is the bordism theory constructed in the same way as $H_{n}^{\chi}(-)$ which uses stratified cycles and bordisms $V$ such that $\operatorname{codim}_{V} S V \geqslant \beta$ and $\operatorname{codim}_{\partial V} S(\partial V) \geqslant \beta$. The same arguments used for $H_{n}^{\chi}(-)$ prove that $H_{n}^{\chi}(-; \beta)$ is a generalized homology theory (defined on the category of all topological pairs). The dimension axiom is not satisfied, but the cone construction gives

$$
\begin{array}{ll}
H_{0}(\text { point } ; \beta) \cong \mathbf{Z}_{2} ; & \\
H_{i}(\text { point } ; \beta)=\eta_{i}, & \text { for } i \leqslant \beta-2 ; \\
H_{i}(\text { point } ; \beta)=0, & \text { for } i>\beta-2 .
\end{array}
$$

In exactly the same fashion one constructs $\bar{H}_{n}^{\chi}(-; \beta)$, the bordism theory of trivialised cycles with singularities in codimension $\geqslant \beta$. There is a natural transformation $F(-): \bar{H}_{n}^{\chi}(-; \beta) \rightarrow H_{n}^{\chi}(-; \beta)$ defined by forgetting the trivialisations. $F$ is clearly a map of homology theories and, by the cone construction, $F$ (point) is an isomorphism. Therefore, by the comparison theorem, $F$ is an isomorphism on finite CW-complexes.

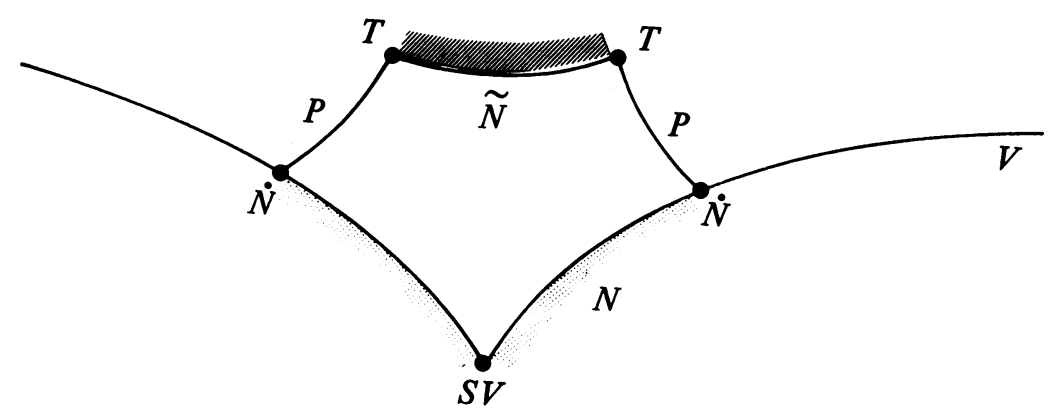

FIGURE 13 
Consider $f: N_{1} \rightarrow N$ with $N_{1}=P \cup \tilde{N}$; here $f: P \rightarrow N$ is a bordism in $H_{n}^{\chi}(N ; \beta)$ between $\dot{N} \subset N$ and a trivialised cycle $T$ (see Figure 13). The existence of $P$ follows from the existence of the isomorphism $F$ above and the fact that $N$ has the homotopy type of a finite CW-complex. To define $\tilde{N}$, first observe that $f: T \rightarrow N$ is certainly null-homologous in $N$ (because $\dot{N} \subset N$ is null-homologous). Then we define $(\tilde{N}, f)$ as a trivialised cycle whose boundary is $(T, f)$. Finally, the union $P \cup \tilde{N}$ is taken along $T$. Now form $\tilde{V}=\overline{V-N} \cup_{\dot{N}} P \cup \tilde{N}$. In general $\tilde{V}$ will not be a blow-up of $V$, because $\operatorname{dim} S \tilde{N}(\leqslant n-2)$ may be greater than $n-\gamma$. Note that, by construction, $\operatorname{dim} S P=n-\beta<n-\gamma$.

In order to construct the blow-up $(\hat{V}, \hat{N})$ required by the theorem, it suffices to show that, if $\tilde{X}=\left\{\tilde{X}_{1}, \ldots, \tilde{X}_{q}\right\}$ are the top-dimensional strata of $S \tilde{N}$ with codim $\tilde{X}_{i}$ $<\gamma+k$ and $\tilde{V}^{\prime} \subset S \tilde{N}$ is the closure of $\tilde{X}$, then there is a blow-up $\left(\tilde{V}_{1}, \tilde{N}_{1}\right)$ of $(\tilde{V}, \tilde{N})$ along $\tilde{V}^{\prime}$ such that $\operatorname{codim} S \tilde{N}_{1}>\operatorname{codim} \tilde{V}^{\prime}$. An iteration of this procedure will lead to the construction of $(\hat{V}, \hat{N})$.

The crucial point is that $\tilde{N}$ is now a trivialised set. Thus Lemma 2.11 applies and we may assume that $\overline{\tilde{X}}_{i}$ is a trivialised cycle (with appropriate link) for $i=1, \ldots, q$. By Lemma 2.12 we only need to prove that $f_{*}\left[\overline{\tilde{X}}_{i}\right]=0$ in $H_{m}(N), m=\operatorname{dim} \overline{\tilde{X}}_{i}$. But if $m>n-\gamma$ this is true because $N$ deformations retracts onto $V^{\prime}$ and $\operatorname{dim} V^{\prime}=n-\gamma$. If $m \leqslant n-\gamma$, then one may apply to $\left(\tilde{N}, \tilde{V}^{\prime}\right)$ exactly the same arguments applied to $\left(\tilde{N}, \tilde{V}^{\prime}\right)$ in the proof of Theorem 2.10. The only difference is that now $E=E\left(\tilde{V}^{\prime}, \tilde{N}\right)$ is a stratified set not necessarily trivialised, but this causes no problem.

If $\vartheta_{n-1}\left(V^{\prime}\right)=0$, then an obvious sufficient condition for $\vartheta_{n-m-1}^{h}\left(V^{\prime}\right)=0$ is the following: there is a cohomology class $\xi_{h}^{n-m-1} \in H^{n-m-1}(N)$ such that

$$
\left(\xi_{h}^{n-m-1} \mid \dot{N}\right) \cap[\dot{N}]=\mu_{n-m-1}^{h}(\dot{N}) \text {. }
$$

We have an interesting special case when $V^{\prime}=S V$, and consequently $\dot{N}$ is a manifold, $\beta>n$, and $\mu_{n-m-1}^{h}(\dot{N})$ are the Poincaré dual of the classes $\sigma_{h}^{m} \in H^{m}(\dot{N})$.

2.14 COROLlaRY. Let $V$ be a compact unbounded stratified set (not necessarily trivialised) such that codim $S V=\gamma$. Then the following statements are equivalent:

(a) all the classes $\vartheta_{n-m-1}^{h}(S V)$ vanish, for each $m=0, \gamma-1, \ldots, \gamma+k-2$ and for each $h=1, \ldots, \operatorname{dim} \eta_{m}$;

(b) there exists a blow-up $f:(\hat{V}, \hat{N}) \rightarrow(V, N)$ of $V$ along $S V$ such that $V$ is trivialised and codim $S \hat{N} \geqslant \gamma+k$.

Here $k$ is an integer such that $1 \leqslant k \leqslant n+1-\gamma$ and in particular, if $k=n+1-\gamma$ (so that $\gamma+k-2=n-1$ ) we obtain a blow-up $\hat{V}$, which is a manifold.

There is a natural notion of blow-up for stratified $n$-sets with boundary and a straightforward extension of Theorem 2.13 to the relative case; this may be used to deal with the uniqueness problem. We omit the details.

Let $j$ be the inclusion of $S V$ in $V$; it is not difficult to prove that $j_{*} \vartheta_{n-m-1}^{h}(S V)=0$ $\forall m, \forall h$; this may be used to give a "resolution" of $V$ up to a degree one map (not necessarily a blow-up).

We conclude this section by considering an "embedded" case (in analogy with [6, $\S 5])$. Let $V$ be a compact unbounded stratified $n$-set, $M$ a manifold and $V \subset M$. We 
say that $V$ is embedded in $M$ if $M$ is a stratified set and $S M=V$. Let $\hat{V}$ be a compact submanifold of $M$ without boundary. We say that $\hat{V}$ is a resolution of $V$ in $M$ if $\hat{V} \cap E(S V, M)=E(S V, V)$. Two resolutions $\hat{V}_{1}$ and $\hat{V}_{2}$ are said to be bordant in $M$ if and only if $\hat{V}_{1} \cap T(S V, M)$ and $\hat{V}_{2} \cap T(S V, M)$ are $L$-equivalent (in the sense of Thom [15]) relative to $\dot{T}(S V, M)$ in $T(S V, M)$.

2.15 Proposition. Let $V$ be a closed trivialised $n$-cycle embedded (as defined above) in the manifold $M$ of dimension $n+1$. If $M$ is also trivialised, there is a resolution $\hat{V}$ of $V$ in $M$; moreover, $\hat{V}$ is unique up to framed bordism in $M$.

Proof. Let $X$ be a top-dimensional stratum of $S V, \operatorname{dim} X=n-\gamma$. Then $L(X, M)$ is a sphere $S^{\gamma}$ and $L(X, V)$ is a framed $(\gamma-1)$-submanifold of $S^{\gamma}$ (by the definition of a trivialised set). In order to "resolve" the stratum $X$ in this context, we only need to see if there is a framed cobordism of $L(X, V)$ to zero in $D^{\gamma+1}$. But this happens if and only if the "Pontryagin map" $f_{X}: S^{\gamma} \rightarrow S^{1}$ is null-homotopic $\left(f_{X}(L(X, V))=*\right)$. As $V$ is a cycle, $\gamma>1$ and $f_{X} \cong *$. Iterating this argument, one is able to resolve all strata of $S V$. Uniqueness may be proved in a similar way.

There are extensions of the above proposition which lead to interesting examples. They are obtained by slightly relaxing the hypothesis $\operatorname{codim}_{V} S V>1$ and the hypothesis of trivialisations. If $\operatorname{codim}_{V} S V=1$, the blow-up $\hat{V}$ does not exist in general. For example, we could take $M=S^{3}=\mathbf{R}^{3} \cup \infty$ and $V$ the union of the unit sphere $S^{2} \subset \mathbf{R}^{3}$ and the 2-disk $\left\{x^{2}+y^{2} \leqslant 1, z=0\right\}$. Whatever trivialisations we choose, there is no resolution of $V$, even if we do not require that $\hat{V}$ is framed or that $\hat{V} \subset M$. However, if $\operatorname{codim}_{V} S V=1$, the above proof shows that the only obstructions to the existence of a framed resolution $\hat{V}$ of $V$ in $M$ are the degrees of the maps $f_{X}: S^{1} \rightarrow S^{1}\left(f_{X}(L(X, V))=*\right)$ which classify the framed cobordism class of $L(X, V)$ in $S^{1}=L(X, M)$, for each $(n-1)$-dimensional stratum $X$ of $S V$. Now suppose that the tubular neighbourhoods of the strata of $V-S V$ in $M$ are arbitrary (thus possibly not trivial, while all the other tubular neighbourhoods are trivialised); in this case we say that $V$ is embedded in $M$ with trivialised singularity. We ask for a resolution $\hat{V}$ of $V$ in $M$, not necessarily framed in $M$.

2.16 Proposition. Let $V$ be a compact unbounded trivialised $n$-set embedded in the $(n+1)$-manifold $M$ with trivialised singularity. Then $V$ admits a resolution $\hat{V}$ in $M$ if and only if $\vartheta_{n-1}(S V)=0$. Moreover, $\hat{V}$ is unique up to bordism in $M$.

Proof. The idea is the same as for Proposition 2.15, to which we refer for notation. The hypothesis $\vartheta_{n-1}(S V)=0$ allows us to deal with the case where $V$ is not a cycle. Let $X$ be a top-dimensional stratum of $S V, \operatorname{dim} X=n-\gamma$. First we assume $\gamma>1$. The difference from the proof of 2.15 is that now $L(X, V)$ is not necessarily framed in $S^{\gamma}$, so that the right space to use, instead of $S^{1}$, is the Thom space $M O(1) \cong K\left(\mathbf{Z}_{2}, 1\right)$. Thus $\gamma>1$ implies that the Thom map $f_{X}: S^{\gamma} \rightarrow M O(1)$ (classifying $L(X, V)$ ) is null-homotopic.

If $\gamma=1$, then the obstructions to the existence of $\hat{V}$ are the homotopy classes of the maps $f_{X}: S^{1} \rightarrow M O(1)$. But $\pi_{1}(M O(1)) \cong \mathbf{Z}_{2}$ and $\vartheta_{n-1}(S V) \in H_{n-1}(S V)$ is represented, by definition, by the map $\dot{\pi}: \dot{N} \rightarrow S V$, where $N=T(S V, V)$. Thus, by 
Proposition $1.10, \vartheta_{n-1}(S V)=0 \Leftrightarrow L(X, V)$ is an even number of points $\Leftrightarrow f_{X}$ is null-homotopic for all such $X$. The uniqueness part is proved by the same arguments.

Observe that, in constructing the resolution $\hat{V}$, the trivialisations of the tubular neighbourhoods of the strata of $S V$ are used in an essential way. In fact, when we replace $V_{X}$ by $X \times \underset{\sim}{W}$ with $W$ an $L$-equivalence of $L(X, V)$ to zero in $D^{\gamma+1}$, we must be sure that the resulting object has trivial tubular neighbourhoods and this is ensured by the compatibility of the trivialisations. Thus, in general, we can not weaken the condition that $S V$ be trivialised in $M$. However, in some cases, the triviality of tubular neighbourhoods is due to topological reasons and need not be assumed. Such a situation occurs, for instance, when $S V$ has contractible strata.

2.17 Proposition. Let $V$ be a compact unbounded stratified $n$-set embedded in the $(n+1)$-manifold $M$. Suppose that each stratum of $S V$ is contractible and $\boldsymbol{\vartheta}_{n-1}(S V)=$ 0 . Then there is a resolution $\hat{V}$ of $V$ in $M$, unique up to bordism in $M$.

The proof is practically the same as the proof of Proposition 2.16.

An interesting example of the situation described in the above proposition is provided by a compatible triangulation of a closed $n$-dimensional analytic set $V$ with singularity $S V$ in an analytic manifold $M$ of dimension $n+1$. Here the contractible strata are the simplexes of $S V[6,6.1]$.

To obtain more examples of resolvable embedded stratified sets we give the definitions below (following [6]). Let $M$ be a manifold and $M_{1}, \ldots, M_{p+1}$ submanifolds of $M$. We say that $M_{1}, \ldots, M_{p+1}$ intersect transversally in $M$ if either $p=0$ or, assuming inductively that $M_{1}, \ldots, M_{p}$ intersect transversally, it happens that, for any subset $\left\{i_{1}, \ldots, i_{k}\right\}$ of $\{1, \ldots, p\}$ the manifold $M_{i_{1}} \cap \cdots \cap M_{i_{k}}$ intersects $M_{p+1}$ transversally (in the smooth category).

Given compact unbounded stratified sets $V_{1}, \ldots, V_{p+1}$ embedded in the manifold $M$, we say that a compact unbounded stratified set $V$ is the transverse intersection of $V_{1}, \ldots, V_{p+1}$ if:

(a) $V_{1}-S V_{1}, \ldots, V_{p+1}-S V_{p+1}$ intersect transversally;

(b) $(V, S V)=\left(V_{1} \cap \cdots \cap V_{p+1}, S V_{1} \cap \cdots \cap S V_{p+1}\right)$.

We say that a compact unbounded stratified set $Q$ is the local transverse intersection of $V_{1}, \ldots, V_{p+1}$ as above if there exists an open neighbourhood $U$ of $Q$ in $M$ such that $Q$ is the transverse intersection of $V_{1} \cap U, \ldots, V_{p+1} \cap U$ in $U$.

2.18 TheOREM. Let $V_{1}, \ldots, V_{k}$ be compact unbounded stratified $n$-sets uniquely resolvable in the $(n+1)$-dimensional manifold $M$; if $V$ is a local transverse intersection of $V_{1}, \ldots, V_{k}$ in $M$, then $V$ is uniquely resolvable in $M$.

Proof. The proof of $[6,6.3]$ easily adapts to our context, thus we omit the details. The idea is very simple: use the hypothesis to choose resolutions $\left\{\hat{V}_{i}^{\prime}\right\}$ of $\left\{V_{i}\right\}$ in $M$; then use the fact that transversality is stable and generic to change $\hat{V}_{i}^{\prime}$ into $\hat{V}_{i}$ so as to obtain that the $\left\{\hat{V}_{i}\right\}$ intersect transversally. Their intersection is then the required resolution $\hat{V}$. 
3. The oriented cases. The same sort of geometric constructions considered in the last section may be applied to oriented cases. Let $G$ be one of the classical Lie groups $S O, U, S U, S p$ : below we define a (trivialised) $G$-stratified set $V$ from a stratified set, essentially by adding a $G$-structure on each stratum, and requiring that all the conditions for $V$ to be a stratified set respect the extra $G$-structure.

Definition. A $G$-stratified set is a stratified set $(V, \chi, \tau)$ in which each stratum has a structure of a $G$-manifold (see [11] for the definition of a $G$-manifold). We refer to $\S 1$ for notation and terminology. If $W \subset V$ are $G$-stratified sets, we say that $W$ is a restriction of $V$ if (a) and (b) are satisfied and also

(c) the $G$-structure on $X$ is the $G$-structure induced by $X^{\prime}$ via the framing of $X$ in $X^{\prime}$.

With the above definition we are able to define $T\left(V^{\prime}, V\right), \dot{T}\left(V^{\prime}, V\right)$ and $E\left(V^{\prime}, V\right)$ as $G$-stratified sets.

An isomorphism of $G$-stratified sets is an isomorphism $f: V \rightarrow V^{\prime}$ of stratified sets satisfying the additional property

(d) $f$ restricts to an isomorphism of $G$-manifolds between $T_{X, Y}$ and $T_{X^{\prime}, Y^{\prime}}$.

The rest of $\S 1$ translates into the present context in an obvious way. In particular, we have the notion of a G-trivialised set, a G-stratified n-cycle (relative or closed) and of a $G$-trivialised $n$-cycle (relative or closed). We also have an associated bordism theory defined in the usual way. The following theorem is proved like Theorem 1.4.

THEOREM. The bordism theory associated with G-stratified cycles (and the bordism theory associated with G-trivialised cycles) is homology theory with integral coefficients.

Let $\Omega_{m}^{G}$ denote the $m$-dimensional bordism group of $G$-manifolds. First of all one fixes a basis of the free part of $\Omega_{m}^{G}$ and polynomials in the characteristic classes (which are now Chern classes, if $G=U$ or $S U$, and $G$-Pontryagin classes, if $G=S O$ or $S p$ ) such that the corresponding matrix of characteristic numbers is a diagonal matrix. This can be done using the existence of $G$-manifolds $M_{k}$ such that $s_{k}\left[M_{k}\right] \neq 0$ (see [9, §16], for $G=U, S O$; [11, Chapter X], for $G=S U$; and [12] for $G=S p$ ).

Then we can proceed as in $\S 2$ to define the obstructions. The main differences are essentially two.

(1) If $G=S U$ or $S p$, the torsion part of $\Omega_{m}^{G}$ is not detected by cohomology characteristic numbers. Thus, for example, in order to prove that the vanishing of the $(n-\gamma+1)$-obstructions implies that the link of a top-dimensional $(n-\gamma)$ stratum $X$ of $V^{\prime}$ is a boundary, we have the following situation:

(a) if $G=U$, we can proceed exactly as in 2.9 , using the obstructions constructed with Chern classes;

(b) if $G=S O$, we can proceed as in 2.9 , using the obstructions constructed with $S O$-Pontryagin classes and Stiefel-Whitney classes, with a little more care, due to the fact that Stiefel-Whitney classes are unoriented homology classes;

(c) if $G=S U$ or $S p$, the same arguments imply only that the link of a top-dimensional stratum $X$ is an element of the torsion part of $\Omega_{m}^{G}$, and we cannot, in general, have a blow-up of $V$ along $V^{\prime}$. However, we can still get a "2-blow-up" of $V$ along $V^{\prime}$. 
(A $k$-blow-up $f:(\hat{V}, \hat{N}) \rightarrow(V, N)$ is defined in the same way as in 2.3 , replacing (b) by the following.

(b') $\overline{\hat{V}-\hat{N}}$ is a disjoint union $V_{1}+\cdots+V_{k}$ and $f$ restricts to isomorphisms $f \mid$ : $V_{i} \rightarrow \overline{V-N}, i=1, \ldots, k$.)

(2) The characteristic numbers are now in $\mathbf{Z}$ instead of $\mathbf{Z}_{2}$. Thus the vanishing of the obstructions implies the vanishing of a multiple of $f_{*}\left[\tilde{\tilde{X}}_{i}\right]$ in $H_{*}\left(V^{\prime}\right)$ (see 2.10); therefore, in general, the vanishing of the obstructions implies only the existence of a $k$-blow-up: in order to have sufficient conditions for the existence of a blow-up (at least in the cases $G=U$ or $S O$ ) of $V$ along $V^{\prime}$, one may add further conditions on the torsion part of the homology of $V^{\prime}$.

The rest of the constructions of $\$ 2$ can be extended with few more technical difficulties. One may note that, from an explicit construction of the basis of the free part of $\Omega_{m}^{G}$, we derive informations about the multiple required in the resolution of the $k$-dimensional strata. Thus, for example, one can prove results of this kind:

THEOREM. Let $V$ be a compact unbounded n-dimensional $G$-stratified set ( $G=U$ or $S O)$ such that, for each $i=0, \ldots, n-1, H_{i}\left(V^{\prime}\right)$ has no $p$ torsion, for each prime $p$ such that

$p<n / 2+1$ (if $G=U)$,

$2<p<n / 2+1$ (if $G=S O)$.

Then there exists a blow-up $(\hat{V}, \hat{N})$ of $(V, N)$ along $V^{\prime}$, with $\hat{V}$ a G-manifold, if and only if all the obstructions $\vartheta_{n-m-1}^{h}\left(V^{\prime}\right)$ vanish, for each $m=0, \ldots, n-1$.

We conclude this section with a discussion of Thom's example related to stratified sets. In [15] Thom defines a class $z \in H_{7}\left(K\left(\mathbf{Z}_{3}, 1\right) \times K\left(\mathbf{Z}_{3}, 1\right)\right)$ not representable by a smooth oriented manifold.

Proposition. Let $V$ be a $G$-stratified cycle representing $z$. Then:

(a) If $G=S O$, there is a blow-up $f: \hat{V} \rightarrow V$ of $V$ along $S V$, with $\hat{V}$ a G-trivialised cycle such that $S V$ consists of exactly one stratum $X$ of dimension two.

(b) If $G=U, S U$, Sp there is a $2^{k}$-blow-up $f: \hat{V} \rightarrow V$, with $\hat{V}$ a G-trivialised set as above.

Proof. Let $G=S O$ and $N=T(S V, V) . \dot{N}$ bounds a $G$-stratified cycle $N$. From the fact that $S V$ has the homotopy type of a finite $\mathrm{CW}$-complex, we deduce that $\dot{N} \subset N$ bounds a $G$-trivialised cycle $\hat{N} \rightarrow N$. Thus we may assume, up to a blow-up, that $V$ is a $G$-trivialised cycle. As $\Omega_{i}^{S O}=0$ for $i \leqslant 3$, we are able to obtain a blow-up of $V$ which is a $G$-trivialised cycle with, singularity in codimension 5 . By a further blow-up, one constructs a $G$-trivialised cycle $\tilde{V}$ such that:

(a) $S \tilde{V}$ consists of exactly one stratum of dimension two;

(b) $L(S \tilde{V}, \tilde{V})=C \mathbf{P}^{2}$ which represents a generator of $\Omega_{4}^{S O}$;

(c) $S \tilde{V}$ is a $G$-trivialised cycle.

This is a consequence of the analogue of Lemma 2.11 and of the fact that $\Omega_{4}^{S O}$ is generated by $C \mathbf{P}^{2}$.

From (c) we have that the singularity of $S \tilde{V}$ consists of a finite set of points $X_{1}, \ldots, X_{q}$. Consider one of these points, say $X_{i} ; L\left(X_{i}, \tilde{V}\right)$ is a 6-dimensional 
$G$-trivialised cycle $W_{i}$ such that $S W_{i}=W_{i} \cap S \tilde{V}$ is a 1-dimensional $G$-manifold with $L\left(S W_{i}, W_{i}\right)=C \mathbf{P}^{2}$. Choose a 2-dimensional $G$-manifold $D_{i}$ such that $\partial D_{i}=S W_{i}$ and put

$$
Q_{i}=\overline{W_{i}-\left(S W_{i} \times c C \mathbf{P}^{2}\right)} \cup D_{i} \times C \mathbf{P}^{2},
$$

the union being taken along $S W_{i} \times C \mathbf{P}^{2} \cdot Q_{i}$ is a 6-dimensional $G$-manifold which bounds because $\Omega_{6}^{S O}=0$. Thus there is a 7-dimensional $G$-manifold $Q_{i}^{\prime}$ with $\partial Q_{i}^{\prime}=$ $Q_{i}$. Then $\partial\left(Q_{i}^{\prime} \cup_{D_{i} \times C \mathbf{P}^{2}}\left(D_{i} \times c C \mathbf{P}^{2}\right)\right)=W_{i}$ and we may replace $c W_{i}$ with

$$
Q_{i}^{\prime} \cup\left(D_{i} \times c C \mathbf{P}^{2}\right) \text {. }
$$

If we iterate this construction for every point $X_{i}$ the resulting $G$-trivialised cycle is the required $\hat{V}$. Note that $S \hat{V}=\cup_{i}\left(E\left(X_{i}, S \tilde{V}\right) \cup_{\partial D_{i}} D_{i}\right)$. This completes the proof in the case $G=S O$.

Let $G=S U, S p$. The argument is essentially the same as in the previous case, the only difference being that some of the groups $\Omega_{i}^{G}$ are nontrivial, for $i=6$ or $i<4$. However, if $\Omega_{i}^{G} \neq 0$, then $\Omega_{i}^{G} \cong \mathbf{Z}_{2}[11,19]$. Thus the method of desingularisation described above may be applied to obtain a $2^{k}$-blow-up $f: \hat{V} \rightarrow V$ satisfying the required conditions. Finally, the case $G=U$ is included in the case $G=S U$. This completes the proof of the proposition.

The above proposition, although stated for a particular $z$, obviously holds for any 7-dimensional homology class.

Corollary. Let $G=S O, U, S U, S p$; the class $z$ of the above proposition is representable by a $G$-trivialised cycle $\hat{V}$ with $S \hat{V}$ consisting of a single two-dimensional stratum.

Proof. Let $[V]=z$ be as in the above proposition. If $G=S O$, then $f: \hat{V} \rightarrow V$ being a blow-up is a degree-one map, thus the result follows. If $G=S U, U, S p$, then $f: \hat{V} \rightarrow V$ has degree $2^{k}$. Therefore $2^{k} z$ is representable by a $G$-trivialised cycle $\hat{V}$ with the required property. But, by the definition of $z$, we have $3 z=0$, which implies $2^{k} z= \pm z$. Thus either $\hat{V}$ or $-\hat{V}$ represents $z$ as required.

\section{REFERENCES}

1. S. Akbulut and H. C. King, Real algebraic structures on some topological spaces, Univ. of Maryland, preprint, 1979.

2. N. A. Baas, On bordism theory of manifolds with singularity, Math. Scand. 33 (1973), 279-302.

3. S. Buoncristiano and M. Dedò, On resolving singularities and relating bordism to homology, Ann. Scuola Norm. Sup. Pisa 7 (1980), 605-624.

4. S. Buoncristiano, C. P. Rourke and B. J. Sanderson, A geometric approach to homology theory, London Math. Soc. Lecture Notes, no. 18, Cambridge Univ. Press, London, 1976.

5. P. E. Conner and E. E. Floyd, Differentiable periodic maps, vol. 33, Springer-Verlag, Berlin and New York, 1964.

6. M. Kato, Topological resolution of singularities, Topology 12 (1973), 355-372.

7. __ Partial Poincaré duality for k-regular spaces and complex algebraic sets, Topology 16 (1977), 33-50.

8. J. Mather, Notes on topological stability, Lecture Notes, Harvard Univ., 1970.

9. J. W. Milnor and J. D. Stasheff, Characteristic classes, Ann. Math. Studies, vol. 76, Princeton Univ. Press, Princeton, N. J., 1974. 
10. D. A. Stone, Stratified polyhedra, Lecture Notes in Math., vol. 252, Springer-Verlag, Berlin and New York, 1972.

11. R. E. Stong, Notes on cobordism theory, Math. Notes, Princeton Univ. Press, Princeton, N. J., 1968.

12. S_ Some remarks on symplectic cobordism, Ann. of Math. 86 (1967), 425-433.

13. D. Sullivan, Singularities in spaces, Proc. Liverpool Singularity Symposium II, Lecture Notes in Math., vol. 209, Springer-Verlag, Berlin and New York, 1971, pp. 196-206.

14. R. Thom, Stratified sets and morphisms: local models, Proc. Liverpool Singularity Symposium I, Lecture Notes in Math., vol. 192, Springer-Verlag, Berlin and New York, 1971.

15. _ Quelques propriétés globales des applications différentiables, Comm. Math. Helv. 28 (1954), 17-86.

16. __ Ensembles et morphismes stratifiés, Bull. Amer. Math. Soc. 75 (1969), 240-284.

17. A. Verona, Homological properties of abstract prestratifications, Rev. Roumaine Math. Pures Appl. 17 (1972), 1109-1121.

18. N. Levitt, Exotic singular structures on spheres, Trans. Amer. Math. Soc. 205 (1975), 371-388.

19. A. Liulevicius, Notes on homotopy of Thom spectra, Amer. J. Math. 86 (1964), 1-16.

DEPARTMENT OF MATHEMATICS, UNIVERSity OF NAPLES, 80134 NAPLES, ITALY

Department of Mathematics, University of Pisa, 56100 Pisa, Italy 